CRYSTALLOGRAPHIC COMMUNICATIONS

ISSN 2056-9890

Received 18 September 2018

Accepted 30 October 2018

Edited by T. J. Prior, University of Hull, England

Keywords: crystal structure; hydrogen bonds; lanthanide; samarium(III).

CCDC reference: 1876239

Supporting information: this article has supporting information at journals.iucr.org/e

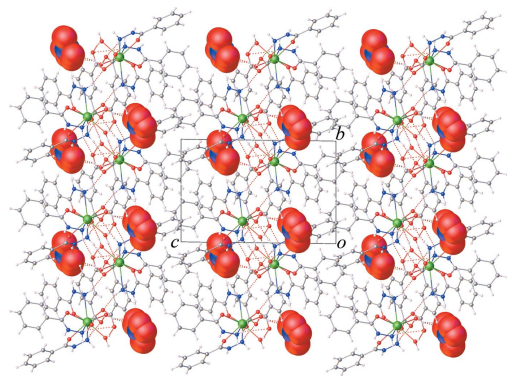

OPEN $\odot$ ACCESS

\section{Crystal structure of diaquatris(benzohydrazide- $\left.\kappa^{2} N, O\right)($ isophthalato- $\kappa O$ )samarium(III) nitrate}

\author{
Chatphorn Theppitak, ${ }^{\mathrm{a}}$ Filip Kielar ${ }^{\mathrm{b}}$ and Kittipong Chainok ${ }^{\mathrm{c} *}$
}

aDivision of Chemistry, Faculty of Science and Technology, Thammasat University, Khlong Luang, Pathum Thani, 12121, Thailand, 'b Department of Chemistry, Faculty of Science, Naresuan University, Muang, Phitsanulok, 65000, Thailand, and ${ }^{\mathrm{C}}$ Materials and Textile Technology, Faculty of Science and Technology, Thammasat University, Khlong Luang, Pathum Thani, 12121, Thailand. *Correspondence e-mail: kc@tu.ac.th

The first benzohydrazide complex of a lanthanide is reported. In the title compound, $\left[\mathrm{Sm}\left(\mathrm{C}_{8} \mathrm{H}_{4} \mathrm{O}_{4}\right)\left(\mathrm{C}_{7} \mathrm{H}_{8} \mathrm{~N}_{2} \mathrm{O}\right)_{3}\left(\mathrm{H}_{2} \mathrm{O}\right)_{2}\right] \mathrm{NO}_{3}$, systematic name diaquatris(benzohydrazide $\left.-\kappa^{2} N, O\right)$ (isophthalato- $\kappa O$ ) samarium(III) nitrate, the $\mathrm{Sm}^{\mathrm{III}}$ ion is nine-coordinated in a distorted tricapped trigonal-prismatic geometry by three oxygen atoms and three nitrogen atoms from three benzhydrazide (bzz) ligands, one oxygen atom from the isophthalate $\left(\mathrm{itp}^{2-}\right)$ ligand, and two oxygen atoms from coordinated water molecules. The nitrate group is disordered over two sets of sites with occupancy factors of 0.310 (17) and 0.690 (17). In the crystal, adjacent molecules are linked into chains via pairs of $\mathrm{O}-\mathrm{H} \cdots \mathrm{O}$ and $\mathrm{N}-\mathrm{H} \cdots \mathrm{O}$ hydrogen bonds between the carboxylate acceptor and the coordinated water and amine $\mathrm{NH}_{2}$ donors. Molecules are further stacked by $\pi-\pi$ interactions involving the benzene ring of the itp ${ }^{2-}$ ligands, forming double chains that extend in the $b$-axis direction. These double chains are further linked into a three-dimensional supramolecular network via hydrogen bonds $(\mathrm{O}-$ $\mathrm{H} \cdots \mathrm{O}, \mathrm{N}-\mathrm{H} \cdots \mathrm{O}$ and $\mathrm{C}-\mathrm{H} \cdots \mathrm{O}$ ) between the complex molecule and the nitrate groups along with $\mathrm{C}-\mathrm{H} \cdots \pi$ and $\pi-\pi$ interactions involving the benzene rings of the bzz and itp ${ }^{2-}$ ligands.

\section{Chemical context}

Research on lanthanide-based coordination compounds is one of the most active fields in chemistry and materials science. Distinct from transition metal centers, lanthanide ions often demonstrate high and variable coordination numbers as well as diverse coordination geometries, which can lead to versatile structures and topologies (Cotton \& Raithby, 2017). They are also very attractive luminescent centers for the high colour purity and relatively long lifetimes arising from electronic transitions within the partially filled $4 f$ orbitals, which make them potential candidates for applications in lighting, photonics and as luminescent probes and sensors (Parker, 2000; Bünzli \& Piguet, 2005; Cui et al., 2018). Besides the metal ions, the organic ligands also have significant effects on the construction of novel lanthanide coordination compounds and their potential applications (Lu et al., 2012; Xu et al., 2016; You et al., 2018). It is well-known that lanthanide ions have a high affinity for and prefer to bind to hard donor atoms such as oxygen-containing organic ligands, for instance aromatic carboxylic acids. Terephthalic acid and its derivatives have thus been widely employed in the synthesis of novel lanthanide-based coordination compounds with interesting architectures and photoluminescence properties (Karmakar et al., 2016; Park \& Oh, 2016). These ligands can exhibit various 
coordination modes when coordinated to the metal centers, as well as serving as antennas or sensitizers to absorb light and transfer energy to the excited states of the central lanthanide ions (Bünzli \& Piguet, 2005). Aromatic organic compounds containing the hydrazide group have been used widely as chemical receptors for sensing anions (Ran et al., 2017; Liu et al., 2018), but have received less attention as metal chelators. In a search for new structural chemistry, we employed benzhydrazide (bzz) and isoterephthalic acid $\left(\mathrm{H}_{2} \mathrm{itp}\right)$ as ligands to react with $\mathrm{Sm}\left(\mathrm{NO}_{3}\right)_{3} \cdot 6 \mathrm{H}_{2} \mathrm{O}$ under hydrothermal conditions, and the crystal structure determination of the title compound is reported herein.

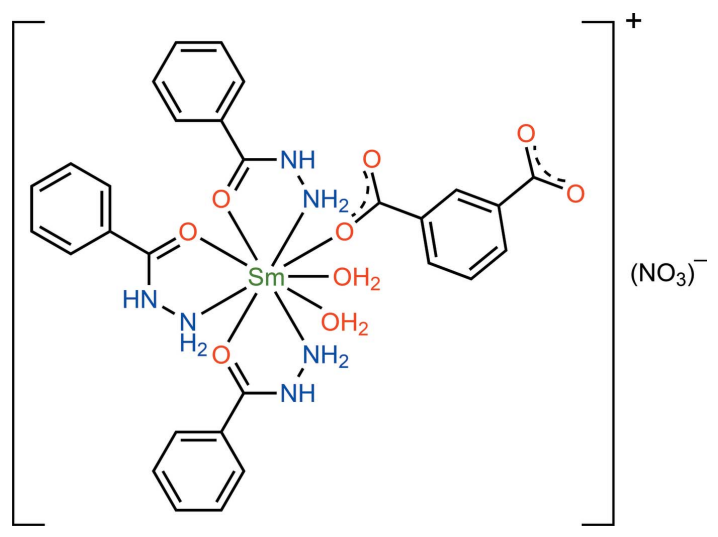

\section{Structural commentary}

The molecular structure of the title compound is shown in Fig. 1. The asymmetric unit comprises one $\mathrm{Sm}^{\mathrm{III}}$ ion, three benzhydrazide (bzz) ligands, one completely deprotonated isophthalate $\left(\mathrm{itp}^{2-}\right.$ ) ligand, two coordinated water molecules, and one disordered $\mathrm{NO}_{3}{ }^{-}$ion. The hydrazide group of the bzz ligand adopts a bidentate $\mu_{2}-\eta^{1}: \eta^{1}$ chelating coordination mode, whereas the carboxylate groups of the fully deprotonated itp ${ }^{2-}$ ligand display a $\mu_{1}-\eta^{1}: \eta^{0}$ monodentate coordina-

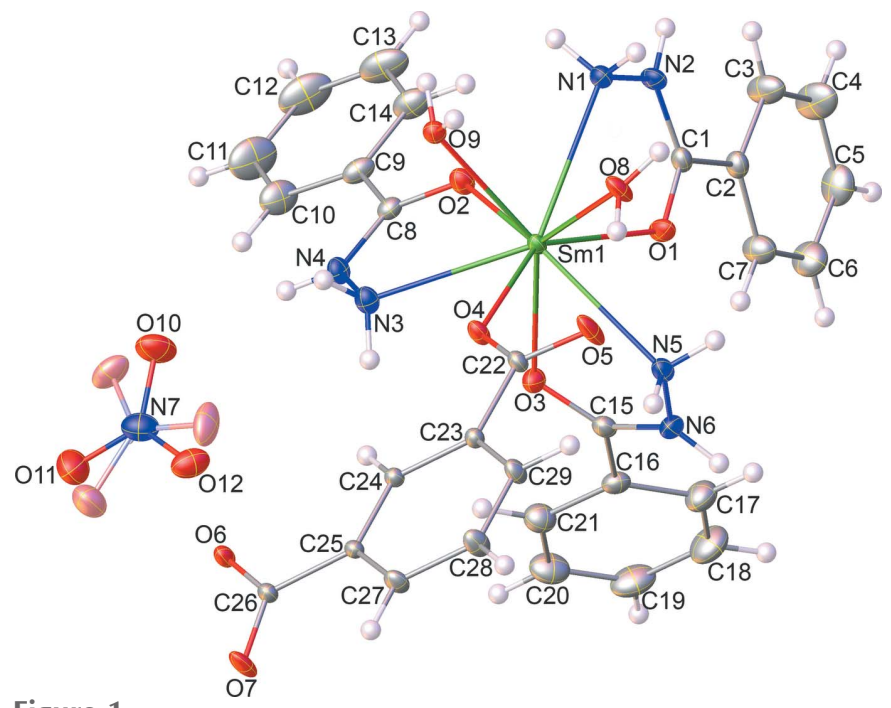

Figure 1

Molecular structure of the title compound, showing the atom-labelling scheme. Displacement ellipsoids are drawn at the $30 \%$ probability level.
Table 1

Hydrogen-bond geometry $\left(\AA,^{\circ}\right)$.

$C g 1$ and $C g 3$ are the centroids of the $\mathrm{C} 2-\mathrm{C} 7$ and $\mathrm{C} 16-\mathrm{C} 21$ rings, respectively.

\begin{tabular}{|c|c|c|c|c|}
\hline$D-\mathrm{H} \cdots A$ & $D-\mathrm{H}$ & $\mathrm{H} \cdots A$ & $D \cdots A$ & $D-\mathrm{H} \cdots A$ \\
\hline $\mathrm{O} 8-\mathrm{H} 8 A \cdots \mathrm{O} 7^{\mathrm{i}}$ & $0.83(2)$ & $1.75(2)$ & $2.574(2)$ & $168(3)$ \\
\hline $\mathrm{O} 8-\mathrm{H} 8 B \cdots \mathrm{O} 5$ & $0.84(2)$ & $1.76(2)$ & $2.585(3)$ & $166(3)$ \\
\hline $\mathrm{O} 9-\mathrm{H} 9 A \cdots \mathrm{O} 12 A^{\mathrm{ii}}$ & $0.83(2)$ & $2.22(2)$ & $2.988(2)$ & $156(3)$ \\
\hline $\mathrm{O} 9-\mathrm{H} 9 A \cdots \mathrm{O} 12 B^{\mathrm{ii}}$ & $0.83(2)$ & $2.09(2)$ & $2.888(2)$ & $162(3)$ \\
\hline $\mathrm{O} 9-\mathrm{H} 9 B \cdots \mathrm{O} 6^{\mathrm{ii}}$ & $0.83(2)$ & $1.81(2)$ & $2.633(2)$ & $170(3)$ \\
\hline $\mathrm{N} 1-\mathrm{H} 1 A \cdots \mathrm{O}^{\mathrm{i}}$ & $0.88(2)$ & $2.14(2)$ & $3.012(3)$ & $172(2)$ \\
\hline $\mathrm{N} 1-\mathrm{H} 1 B \cdots \mathrm{O} 6^{\mathrm{ii}}$ & $0.88(2)$ & $2.14(2)$ & $2.965(3)$ & $156(3)$ \\
\hline $\mathrm{N} 2-\mathrm{H} 2 \cdots \mathrm{O} 11 A^{\mathrm{i}}$ & $0.87(2)$ & $2.22(3)$ & $2.936(2)$ & $140(2)$ \\
\hline $\mathrm{N} 2-\mathrm{H} 2 \cdots \mathrm{O} 12 B^{\mathrm{i}}$ & $0.87(2)$ & $2.10(2)$ & $2.946(3)$ & $164(3)$ \\
\hline $\mathrm{N} 3-\mathrm{H} 3 B \cdots \mathrm{O} 4^{\mathrm{ii}}$ & $0.88(2)$ & $2.53(2)$ & $3.344(3)$ & $155(3)$ \\
\hline $\mathrm{N} 4-\mathrm{H} 4 \cdots \mathrm{O} 10 B$ & $0.87(2)$ & $2.18(2)$ & $3.040(3)$ & $176(3)$ \\
\hline $\mathrm{N} 4-\mathrm{H} 4 \cdots \mathrm{O} 12 A$ & $0.87(2)$ & $2.31(3)$ & $2.970(2)$ & $133(3)$ \\
\hline $\mathrm{N} 5-\mathrm{H} 5 A \cdots \mathrm{O} 5^{\mathrm{iii}}$ & $0.88(2)$ & $2.07(2)$ & $2.878(3)$ & $152(2)$ \\
\hline $\mathrm{N} 5-\mathrm{H} 5 B \cdots \mathrm{O} 5$ & $0.87(2)$ & $2.67(2)$ & $3.199(3)$ & $120(2)$ \\
\hline $\mathrm{N} 5-\mathrm{H} 5 B \cdots \mathrm{O} 7^{\mathrm{iv}}$ & $0.87(2)$ & $2.15(2)$ & $2.940(3)$ & $151(2)$ \\
\hline $\mathrm{N} 6-\mathrm{H} 6 \cdots \mathrm{O} 10 A^{\mathrm{v}}$ & $0.87(2)$ & $2.22(3)$ & $2.961(2)$ & $142(2)$ \\
\hline $\mathrm{N} 6-\mathrm{H} 6 \cdots \mathrm{O} 11 B^{\mathrm{v}}$ & $0.87(2)$ & $2.28(2)$ & $3.115(3)$ & $159(3)$ \\
\hline $\mathrm{C} 10-\mathrm{H} 10 \cdots \mathrm{O} 10 B$ & 0.93 & 2.36 & $3.282(2)$ & 173 \\
\hline $\mathrm{C} 11-\mathrm{H} 11 \cdots C g 1^{\mathrm{vi}}$ & 0.93 & 3.10 & $3.866(2)$ & 137 \\
\hline $\mathrm{C} 13-\mathrm{H} 13 \cdots C g 3^{\mathrm{vii}}$ & 0.93 & 3.02 & $3.712(4)$ & 132 \\
\hline
\end{tabular}

Symmetry codes: (i) $x, y-1, z$; (ii) $-x+1,-y+1,-z+1$; (iii) $-x+2,-y,-z+1$; (iv) $-x+2,-y+1,-z+1$; (v) $x+1, y-1, z$; (vi) $x-1, y+1, z$; (vii) $x-1, y, z$.

tion fashion. The $\mathrm{Sm}^{\mathrm{III}}$ ion is nine-coordinated by three oxygen atoms $(\mathrm{O} 1, \mathrm{O} 2, \mathrm{O} 3)$ and three nitrogen atoms $(\mathrm{N} 1, \mathrm{~N} 3$, $\mathrm{N} 5)$ of three different bzz ligands, one oxygen atom (O4) from the completely deprotonated itp $^{2-}$ ligand, and other two oxygen atoms $(\mathrm{O} 8, \mathrm{O} 9)$ from the coordinated water molecules. The central metal $\mathrm{Sm}^{\text {III }}$ atom can be described as having a distorted tricapped trigonal-prismatic geometry, Fig. 2, with the $\mathrm{Sm}-\mathrm{N}$ and the $\mathrm{Sm}-\mathrm{O}$ bond lengths in the ligand ranging from 2.633 (2) to 2.694 (2) $\AA$ and 2.340 (2) to 2.478 (2) $\AA$, respectively, and the $\mathrm{N} / \mathrm{O}-\mathrm{Sm}-\mathrm{N} / \mathrm{O}$ bond angles fall in the range 60.97 (6) to $145.24(6)^{\circ}$. These values are comparable to other reported values for oxygen/nitrogen-coordinated $\mathrm{Sm}^{\text {III }}$ complexes (Alipour et al., 2016; An et al., 2016).

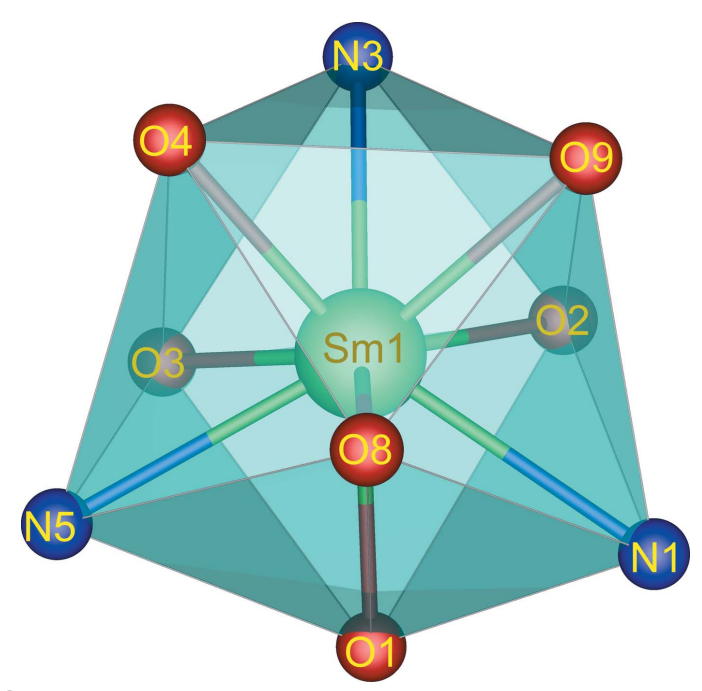

Figure 2

View of the distorted tricapped trigonal-prismatic coordination geometry of the central $\mathrm{Sm}^{\mathrm{III}}$ atom. 


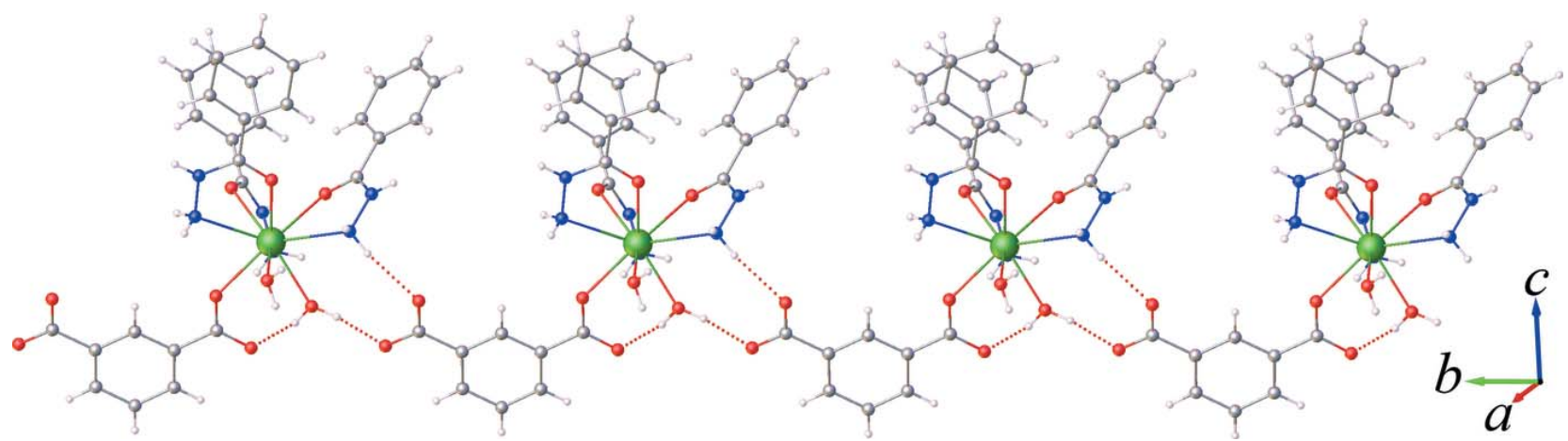

Figure 3

View of a supramolecular chain formed by $\mathrm{O}-\mathrm{H} \cdots \mathrm{O}$ and $\mathrm{N}-\mathrm{H} \cdots \mathrm{O}$ hydrogen bonds.

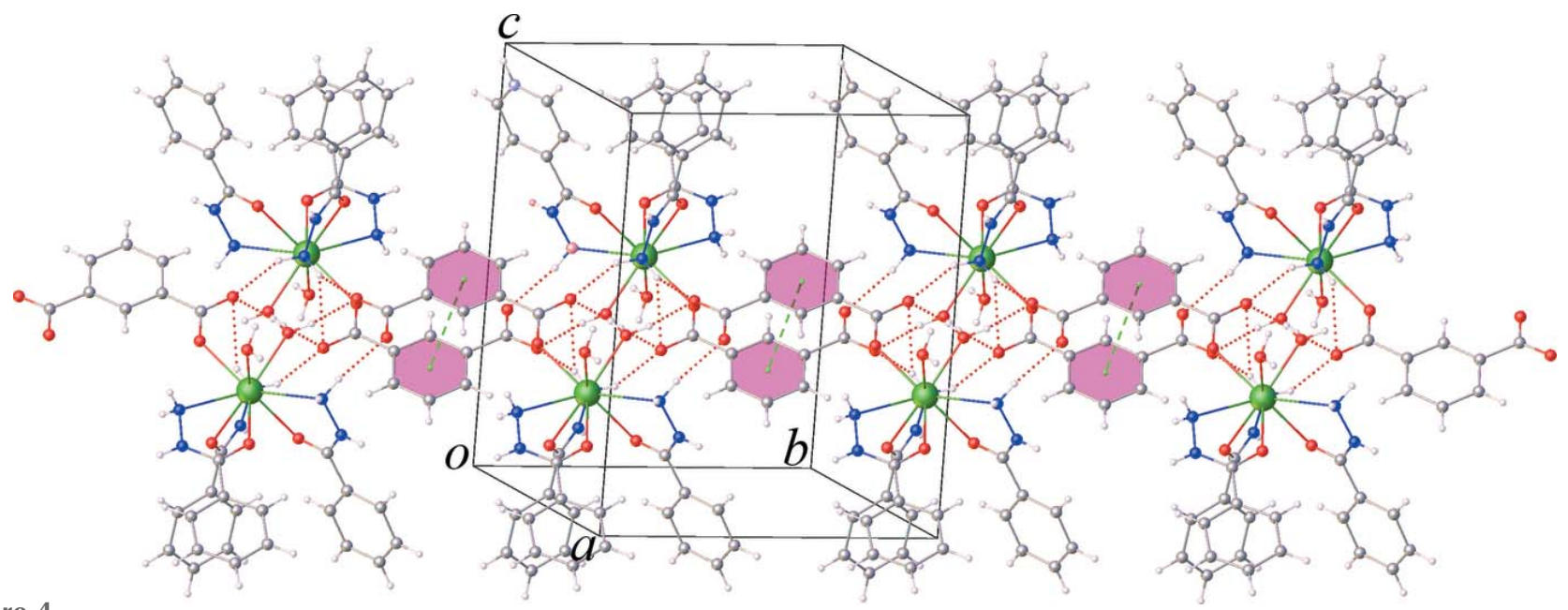

Figure 4

View of a supramolecular double chain sustained by $\mathrm{O}-\mathrm{H} \cdots \mathrm{O}$ and $\mathrm{N}-\mathrm{H} \cdots \mathrm{O}$ hydrogen bonding along with $\pi-\pi$ stacking interactions.

\section{Supramolecular features}

As can be seen in Fig. 3, one carboxylate group of the itp ${ }^{2-}$ ligand adopts a monodentate coordination mode to the $\mathrm{Sm}^{\text {III }}$ ion, while the other acts as an acceptor of hydrogen-bonding interactions (Table 1) involving the water molecules and the amine $\mathrm{NH}_{2}$ group of the bzz ligand of an adjacent complex molecules. This arrangement gives rise to chains extending along the $b$-axis direction by offset $\pi-\pi$ interactions between the benzene rings of symmetry-related itp ${ }^{2-}$ ligands, Fig. 4, with a centroid to centroid distance of $\mathrm{Cg} 4 \cdots \mathrm{Cg} 4^{\mathrm{i}}=$ 3.692 (2) $\AA$ and a dihedral angle $=0.0(2)^{\circ}$ [ $C g 4$ is the centroid of the C23-C29 ring; symmetry code: (i) $2-x, 1-y, 1-z$ ). Fig. 5 shows the crystal packing of the title compound along the $a$ axis. The three-dimensional supramolecular architecture of the crystal is sustained by numerous $\mathrm{O}-\mathrm{H} \cdots \mathrm{O}, \mathrm{N}-\mathrm{H} \cdots \mathrm{O}$ and $\mathrm{C}-\mathrm{H} \cdots \mathrm{O}$ hydrogen bonds between the complex molecules and the nitrate groups along with weak $\mathrm{C}-\mathrm{H} \cdots \pi$ interactions between the aromatic $\mathrm{C}-\mathrm{H}$ bonds and the benzene rings of the bzz ligands, Table 1 . Furthermore, weak aromatic $\pi-\pi$ stacking interactions involving the bzz ligands $\left[C g 1 \cdots C g 1^{\mathrm{ii}}=3.882(2) \AA\right.$, dihedral angle $=0.0(5)^{\circ} ; C g 1$ is the centroid of the $\mathrm{C} 2-\mathrm{C} 7$ ring; symmetry code: (ii) $1-x,-y$, $2-z ;]$, and the bzz and itp ${ }^{2-}$ ligands $\left[C g 2 \cdots C g 4^{4 i i}=\right.$ $3.715(3) \AA$, dihedral angle $=4.7(9)^{\circ} ; C g 2$ is the centroid of the C9-C14 ring; symmetry code: (iii) $1-x, 1-y, 1-z]$, are also observed, which help further to stabilize the crystal structure.

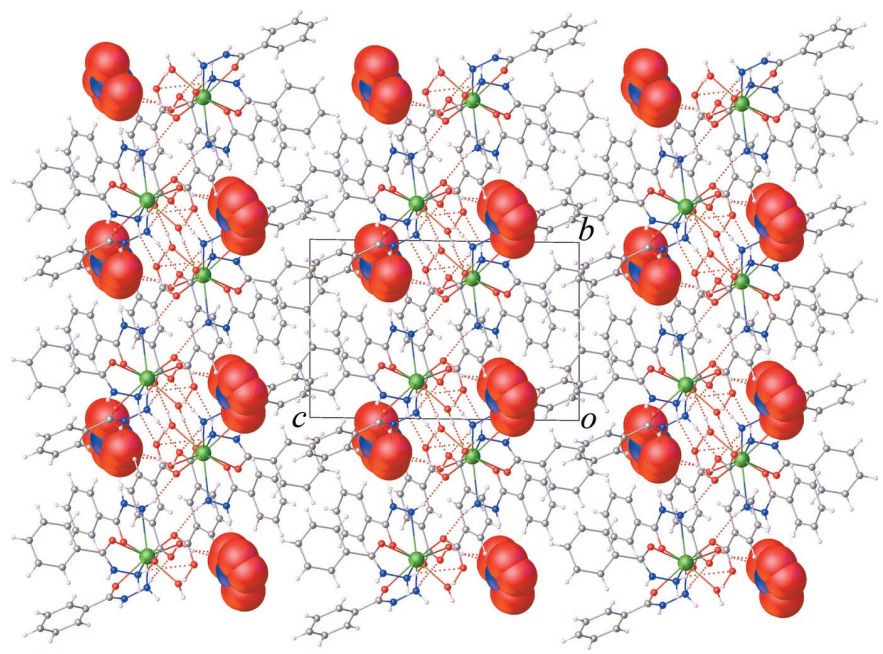

Figure 5

The crystal packing of the title compound, viewed along the $a$ axis. The nitrate molecules are shown with a space-filling model. 
Table 2

Experimental details.

\begin{tabular}{|c|c|}
\hline \multicolumn{2}{|l|}{ Crystal data } \\
\hline Chemical formula & $\begin{array}{l}{\left[\mathrm{Sm}\left(\mathrm{C}_{8} \mathrm{H}_{4} \mathrm{O}_{4}\right)\left(\mathrm{C}_{7} \mathrm{H}_{8} \mathrm{~N}_{2} \mathrm{O}\right)_{3}\left(\mathrm{H}_{2} \mathrm{O}\right)_{2}\right]-} \\
\quad \mathrm{NO}_{3}\end{array}$ \\
\hline$M_{\mathrm{r}}$ & 820.96 \\
\hline Crystal system, space group & Triclinic, $P \overline{1}$ \\
\hline Temperature (K) & 296 \\
\hline$a, b, c(\AA)$ & $\begin{array}{l}11.0784(12), 11.2518(13), \\
\quad 15.3590(18)\end{array}$ \\
\hline$\alpha, \beta, \gamma\left({ }^{\circ}\right)$ & $84.039(4), 78.487(4), 62.042(3)$ \\
\hline$V\left(\AA^{3}\right)$ & $1656.9(3)$ \\
\hline$Z$ & 2 \\
\hline Radiation type & Мо $K \alpha$ \\
\hline$\mu\left(\mathrm{mm}^{-1}\right)$ & 1.84 \\
\hline Crystal size $(\mathrm{mm})$ & $0.22 \times 0.2 \times 0.2$ \\
\hline \multicolumn{2}{|l|}{ Data collection } \\
\hline Diffractometer & Bruker D8 QUEST CMOS \\
\hline Absorption correction & $\begin{array}{l}\text { Multi-scan (SADABS; Bruker, } \\
\text { 2016) }\end{array}$ \\
\hline$T_{\min }, T_{\max }$ & $0.698,0.746$ \\
\hline $\begin{array}{l}\text { No. of measured, independent and } \\
\text { observed }[I>2 \sigma(I)] \text { reflections }\end{array}$ & $52341,7637,6577$ \\
\hline$R_{\text {int }}$ & 0.056 \\
\hline$(\sin \theta / \lambda)_{\max }\left(\AA^{-1}\right)$ & 0.652 \\
\hline \multicolumn{2}{|l|}{ Refinement } \\
\hline$R\left[F^{2}>2 \sigma\left(F^{2}\right)\right], w R\left(F^{2}\right), S$ & $0.027,0.053,1.05$ \\
\hline No. of reflections & 7637 \\
\hline No. of parameters & 531 \\
\hline No. of restraints & 55 \\
\hline $\mathrm{H}$-atom treatment & $\begin{array}{l}\mathrm{H} \text { atoms treated by a mixture of } \\
\text { independent and constrained } \\
\text { refinement }\end{array}$ \\
\hline$\Delta \rho_{\max }, \Delta \rho_{\min }\left(\mathrm{e} \AA^{-3}\right)$ & $0.47,-0.36$ \\
\hline
\end{tabular}

Computer programs: APEX3 and SAINT (Bruker, 2016), SHELXT (Sheldrick, 2015a), SHELXL2014 (Sheldrick, 2015b) and OLEX2 (Dolomanov et al., 2009).

\section{Database survey}

A search of the Cambridge Structural Database (CSD, version 5.39, last update August 2018; Groom et al., 2016) gave 20 hits for the benzohydrazide complexes with transition metal ions, but none of them involves a lanthanide ion. The most typical coordination mode of benzohydrazide ligands in structures appears to be a bidentate chelating mode with metal centers through nitrogen and oxygen donor atoms (BOHYCU, Nyburg et al., 1971; EKAMIM, Odunola et al., 2003; EZARED, EZARIH, Patel et al., 2011; XUQYUD01, Thiam et al., 2009). In these complexes, the nitrogen atoms of the hydrazide group serve as donors for hydrogen bonding.

\section{Synthesis and crystallization}

A mixture of $\mathrm{Sm}\left(\mathrm{NO}_{3}\right)_{3} \cdot 6 \mathrm{H}_{2} \mathrm{O}$ (44.5 mg, $\left.0.1 \mathrm{mmol}\right)$, bzz (27.4 mg, $0.2 \mathrm{mmol}), \mathrm{H}_{2}$ itp $(16.5 \mathrm{mg}, 0.1 \mathrm{mmol})$, and $\mathrm{H}_{2} \mathrm{O}$ $(4 \mathrm{ml})$ was sealed in a $15 \mathrm{ml}$ Teflon-lined steel autoclave and heated at $373 \mathrm{~K}$ for $24 \mathrm{~h}$. The mixture was cooled to room temperature and light-yellow block-shaped crystals of the title compound were obtained in $79 \%$ yield $(35.2 \mathrm{mg}$, based on $\mathrm{Sm}^{\text {III }}$ source). Analysis calculated (\%) for $\mathrm{C}_{29} \mathrm{H}_{32} \mathrm{~N}_{7} \mathrm{O}_{12} \mathrm{Sm}$ (1376.80): C 42.43; H 3.93; N 11.94\%. Found: C 42.46; H 3.96; N $11.90 \%$.

\section{Refinement}

Crystal data, data collection and structure refinement details are summarized in Table 2. All $\mathrm{H}$ atoms were located in difference maps. The $\mathrm{H}$ atoms bonded to $\mathrm{C}$ atoms were treated as riding atoms in geometrically idealized position with $\mathrm{C}-\mathrm{H}$ distances of $0.93 \AA$ and with $U_{\text {iso }}(\mathrm{H})=1.2 U_{\text {eq }}(\mathrm{C})$. The $\mathrm{H}$ atoms bonded to $\mathrm{O}$ and $\mathrm{N}$ atoms were located in a difference-Fourier map, but were refined with distance restraints of $\mathrm{O}-\mathrm{H}=0.84$ $\pm 0.01 \AA$ and $\mathrm{N}-\mathrm{H}=0.88 \pm 0.01 \AA$, and with $U_{\text {iso }}(\mathrm{H})=$ $1.5 U_{\text {eq }}(\mathrm{O})$ and $1.2 U_{\text {eq }}(\mathrm{N})$. The nitrate group is disordered over two sets of sites, with occupancy factors of 0.310 (17) and 0.690 (17).

\section{Funding information}

The authors acknowledge financial support provided from the NRCT and administered by the Division of Research Administration at Thammasat University under grant No. 25/ 2560. This work was also partially supported by the Thailand Research Fund under grant No. RTA6180007.

\section{References}

Alipour, M., Akintola, O., Buchholz, A., Mirzaei, M., EshtiaghHosseini, H., Görls, H. \& Plass, W. (2016). Eur. J. Inorg. Chem. pp. 5356-5365.

An, R., Zhao, H., Hu, H.-M., Wang, X., Yang, M.-L. \& Xue, G. (2016). Inorg. Chem. 55, 871-876.

Bruker (2016). APEX3, SAINT and SADABS. Bruker AXS Inc., Madison, Wisconsin, USA.

Bünzli, J. G. \& Piguet, C. (2005). Chem. Soc. Rev. 34, 1048-1077.

Cotton, S. A. \& Raithby, P. R. (2017). Coord. Chem. Rev. 340, 220 231.

Cui, Y., Zhang, J., He, H. \& Qian, G. (2018). Chem. Soc. Rev. 47, 5740-5785.

Dolomanov, O. V., Bourhis, L. J., Gildea, R. J., Howard, J. A. K. \& Puschmann, H. (2009). J. Appl. Cryst. 42, 339-341.

Groom, C. R., Bruno, I. J., Lightfoot, M. P. \& Ward, S. C. (2016). Acta Cryst. B72, 171-179.

Karmakar, A., Hazra, S., Guedes da Silva, M. F. C., Paul, A. \& Pombeiro, A. J. L. (2016). CrystEngComm, 18, 1337-1349.

Liu, F., Fan, C., Tu, Y. \& Pu, S. (2018). RSC Adv. 8, 31113-31120.

Lu, W.-G., Zhong, D.-C., Jiang, L. \& Lu, T.-B. (2012). Cryst. Growth Des. 12, 3675-3683.

Nyburg, S. C., Baker, R. J. \& Szymanski, J. T. (1971). Inorg. Chem. 10, 138-146.

Odunola, O. A., Adeoye, I. O., Woods, J. A. O. \& Gelebe, A. C. (2003). Synth. React. Inorg. Met.-Org. Chem. 33, 205-221.

Park, J. \& Oh, M. (2016). CrystEngComm, 18, 8372-8376.

Parker, D. (2000). Coord. Chem. Rev. 205, 109-130.

Patel, R. N., Singh, A., Shukla, K. K., Patel, D. K. \& Sondhiya, V. P. (2011). Transition Met. Chem. 36, 179-187.

Ran, X., Gao, Q., Zhang, Y. \& Guo, L. (2017). RSC Adv. 7, 5601656022.

Sheldrick, G. M. (2015a). Acta Cryst. A71, 3-8.

Sheldrick, G. M. (2015b). Acta Cryst. C71, 3-8.

Thiam, E. I., Barry, A. H., Navaza, A., Retailleau, P., Gaye, M. \& Sall, A. S. (2009). Acta Cryst. E65, m1014.

Xu, H., Cao, C.-S., Kang, X. M. \& Zhao, B. (2016). Dalton Trans. 45, 18003-18017.

You, L.-X., Zhao, B.-B., Liu, H.-J., Wang, S.-J., Xiong, G., He, Y.-K., Ding, F., Joos, J. J., Smet, P. F. \& Sun, Y.-G. (2018). CrystEngComm, 20, 615-623. 


\section{supporting information}

Acta Cryst. (2018). E74, 1691-1694 [https://doi.org/10.1107/S2056989018015360]

\section{Crystal structure of diaquatris(benzohydrazide- $\kappa^{2} N, O$ ) (isophthalato- $\kappa O)$ samarium(III) nitrate}

\section{Chatphorn Theppitak, Filip Kielar and Kittipong Chainok}

Computing details

Data collection: APEX3 (Bruker, 2016); cell refinement: SAINT (Bruker, 2016); data reduction: SAINT (Bruker, 2016); program(s) used to solve structure: SHELXT (Sheldrick, 2015a); program(s) used to refine structure: SHELXL2014 (Sheldrick, 2015b); molecular graphics: OLEX2 (Dolomanov et al., 2009); software used to prepare material for publication: OLEX2 (Dolomanov et al., 2009).

Diaquatris(benzohydrazide- $\kappa^{2} \mathrm{~N}, \mathrm{O}$ ) (isophthalato- $\kappa \mathrm{O}$ ) samarium(III) nitrate

Crystal data

$\left[\mathrm{Sm}\left(\mathrm{C}_{8} \mathrm{H}_{4} \mathrm{O}_{4}\right)\left(\mathrm{C}_{7} \mathrm{H}_{8} \mathrm{~N}_{2} \mathrm{O}\right)_{3}\left(\mathrm{H}_{2} \mathrm{O}\right)_{2}\right] \mathrm{NO}_{3}$

$M_{r}=820.96$

Triclinic, $P \overline{1}$

$a=11.0784(12) \AA$

$b=11.2518(13) \AA$

$c=15.3590(18) \AA$

$\alpha=84.039(4)^{\circ}$

$\beta=78.487(4)^{\circ}$

$\gamma=62.042(3)^{\circ}$

$V=1656.9(3) \AA^{3}$

\section{Data collection}

Bruker D8 QUEST CMOS diffractometer

Radiation source: microfocus sealed x-ray tube, Incoatec I $\mu$ us

GraphiteDouble Bounce Multilayer Mirror monochromator

Detector resolution: 10.5 pixels $\mathrm{mm}^{-1}$ $\varphi$ and $\omega$ scans

Absorption correction: multi-scan

(SADABS; Bruker, 2016)

Refinement

Refinement on $F^{2}$

Least-squares matrix: full

$R\left[F^{2}>2 \sigma\left(F^{2}\right)\right]=0.027$

$w R\left(F^{2}\right)=0.053$

$S=1.05$

7637 reflections

531 parameters
$Z=2$

$F(000)=826$

$D_{\mathrm{x}}=1.645 \mathrm{Mg} \mathrm{m}^{-3}$

Mo $K \alpha$ radiation, $\lambda=0.71073 \AA$

Cell parameters from 9800 reflections

$\theta=3.1-27.3^{\circ}$

$\mu=1.84 \mathrm{~mm}^{-1}$

$T=296 \mathrm{~K}$

Block, light yellow

$0.22 \times 0.2 \times 0.2 \mathrm{~mm}$

$T_{\min }=0.698, T_{\max }=0.746$

52341 measured reflections

7637 independent reflections

6577 reflections with $I>2 \sigma(I)$

$R_{\text {int }}=0.056$

$\theta_{\max }=27.6^{\circ}, \theta_{\min }=3.1^{\circ}$

$h=-14 \rightarrow 14$

$k=-14 \rightarrow 14$

$l=-19 \rightarrow 20$

55 restraints

Primary atom site location: dual

Hydrogen site location: mixed

$\mathrm{H}$ atoms treated by a mixture of independent and constrained refinement

$w=1 /\left[\sigma^{2}\left(F_{\mathrm{o}}^{2}\right)+(0.0209 P)^{2}+0.7036 P\right]$

where $P=\left(F_{\mathrm{o}}^{2}+2 F_{\mathrm{c}}^{2}\right) / 3$ 
$(\Delta / \sigma)_{\max }=0.001$

$\Delta \rho_{\min }=-0.36$ e $\AA^{-3}$

$\Delta \rho_{\max }=0.47 \mathrm{e} \AA^{-3}$

Special details

Geometry. All esds (except the esd in the dihedral angle between two 1.s. planes) are estimated using the full covariance matrix. The cell esds are taken into account individually in the estimation of esds in distances, angles and torsion angles; correlations between esds in cell parameters are only used when they are defined by crystal symmetry. An approximate (isotropic) treatment of cell esds is used for estimating esds involving l.s. planes.

Fractional atomic coordinates and isotropic or equivalent isotropic displacement parameters $\left(\AA^{2}\right)$

\begin{tabular}{|c|c|c|c|c|c|}
\hline & $x$ & $y$ & $z$ & $U_{\text {iso }} * / U_{\text {eq }}$ & Occ. $(<1)$ \\
\hline Sm1 & $0.64247(2)$ & $0.20819(2)$ & $0.60370(2)$ & $0.02461(4)$ & \\
\hline $\mathrm{O} 1$ & $0.68088(19)$ & $0.03820(18)$ & $0.72193(12)$ & $0.0396(4)$ & \\
\hline $\mathrm{O} 2$ & $0.41572(18)$ & $0.28637(17)$ & $0.69227(12)$ & $0.0384(4)$ & \\
\hline $\mathrm{O} 3$ & $0.67732(18)$ & $0.29498(17)$ & $0.73343(12)$ & $0.0368(4)$ & \\
\hline $\mathrm{O} 4$ & $0.74767(17)$ & $0.32614(15)$ & $0.50425(11)$ & $0.0320(4)$ & \\
\hline O5 & $0.93740(19)$ & $0.16420(17)$ & $0.43030(15)$ & $0.0507(5)$ & \\
\hline O6 & $0.69780(16)$ & $0.79297(15)$ & $0.48404(12)$ & $0.0337(4)$ & \\
\hline $\mathrm{O} 7$ & $0.88238(19)$ & $0.81877(17)$ & $0.42352(14)$ & $0.0466(5)$ & \\
\hline O8 & $0.7757(2)$ & $0.05576(18)$ & $0.48831(14)$ & $0.0409(5)$ & \\
\hline H8A & $0.799(3)$ & $-0.0206(17)$ & $0.470(2)$ & $0.069(11)^{*}$ & \\
\hline $\mathrm{H} 8 \mathrm{~B}$ & $0.836(3)$ & $0.079(3)$ & $0.465(2)$ & $0.075(12)^{*}$ & \\
\hline O9 & $0.50028(18)$ & $0.28060(17)$ & $0.48404(12)$ & $0.0316(4)$ & \\
\hline H9A & $0.548(2)$ & $0.243(3)$ & $0.4371(11)$ & $0.036(8)^{*}$ & \\
\hline H9B & $0.431(2)$ & $0.267(3)$ & $0.493(2)$ & $0.055(10)^{*}$ & \\
\hline O10A & $0.1374(12)$ & $0.878(2)$ & $0.7680(9)$ & $0.066(5)$ & $0.310(17)$ \\
\hline O10B & $0.1940(8)$ & $0.8003(8)$ & $0.7345(4)$ & $0.0735(19)$ & $0.690(17)$ \\
\hline O11A & $0.266(2)$ & $0.9732(16)$ & $0.7463(14)$ & $0.088(5)$ & $0.310(17)$ \\
\hline O11B & $0.2010(9)$ & $0.9791(7)$ & $0.7585(5)$ & $0.079(2)$ & $0.690(17)$ \\
\hline $\mathrm{O} 12 \mathrm{~A}$ & $0.357(2)$ & $0.7680(15)$ & $0.7052(12)$ & $0.076(5)$ & $0.310(17)$ \\
\hline O12B & $0.3861(6)$ & $0.8098(8)$ & $0.6970(5)$ & $0.0613(16)$ & $0.690(17)$ \\
\hline N1 & $0.5415(2)$ & $0.0317(2)$ & $0.60532(14)$ & $0.0312(5)$ & \\
\hline $\mathrm{H} 1 \mathrm{~A}$ & $0.589(2)$ & $-0.0320(19)$ & $0.5659(13)$ & $0.036(8)^{*}$ & \\
\hline H1B & $0.4578(15)$ & $0.074(3)$ & $0.5919(19)$ & $0.048(9)^{*}$ & \\
\hline N2 & $0.5377(2)$ & $-0.0322(2)$ & $0.68934(14)$ & $0.0347(5)$ & \\
\hline $\mathrm{H} 2$ & $0.487(2)$ & $-0.073(2)$ & $0.7027(18)$ & $0.042(8)^{*}$ & \\
\hline N3 & $0.4835(2)$ & $0.4741(2)$ & $0.62389(15)$ & $0.0358(5)$ & \\
\hline $\mathrm{H} 3 \mathrm{~A}$ & $0.534(3)$ & $0.503(3)$ & $0.6428(18)$ & $0.047(9)^{*}$ & \\
\hline $\mathrm{H} 3 \mathrm{~B}$ & $0.448(3)$ & $0.520(3)$ & $0.5775(14)$ & $0.062(10)^{*}$ & \\
\hline N4 & $0.3651(2)$ & $0.5034(2)$ & $0.68984(14)$ & $0.0331(5)$ & \\
\hline $\mathrm{H} 4$ & $0.313(3)$ & $0.5874(13)$ & 0.7034 (19) & $0.050(9)^{*}$ & \\
\hline N5 & $0.8956(2)$ & $0.1034(2)$ & $0.63928(15)$ & $0.0318(5)$ & \\
\hline $\mathrm{H} 5 \mathrm{~A}$ & $0.930(3)$ & $0.0158(11)$ & $0.6346(18)$ & $0.040(8)^{*}$ & \\
\hline $\mathrm{H} 5 \mathrm{~B}$ & $0.949(2)$ & $0.131(3)$ & $0.6035(15)$ & $0.037(8)^{*}$ & \\
\hline N6 & $0.8946(2)$ & $0.1288(2)$ & $0.72747(15)$ & $0.0356(5)$ & \\
\hline H6 & $0.9703(18)$ & $0.084(2)$ & $0.7502(17)$ & $0.044(8) *$ & \\
\hline N7A & $0.2522(11)$ & $0.8721(11)$ & $0.7372(13)$ & $0.032(4)$ & $0.310(17)$ \\
\hline N7B & $0.2615(8)$ & $0.8629(9)$ & $0.7303(8)$ & $0.056(2)$ & $0.690(17)$ \\
\hline
\end{tabular}




\begin{tabular}{|c|c|c|c|c|}
\hline $\mathrm{C} 1$ & $0.6114(3)$ & $-0.0239(2)$ & $0.74407(17)$ & $0.0325(6)$ \\
\hline $\mathrm{C} 2$ & $0.6105(3)$ & -0.0903 & $0.83308(18)$ & $0.0368(6)$ \\
\hline $\mathrm{C} 3$ & $0.5432(4)$ & $-0.1672(4)$ & $0.8608(2)$ & $0.0667(10)$ \\
\hline $\mathrm{H} 3$ & 0.495609 & -0.181394 & 0.822857 & $0.080 *$ \\
\hline $\mathrm{C} 4$ & $0.5465(5)$ & $-0.2234(4)$ & $0.9454(3)$ & $0.0846(13)$ \\
\hline $\mathrm{H} 4 \mathrm{~A}$ & 0.500847 & -0.275456 & 0.963900 & $0.101 *$ \\
\hline $\mathrm{C} 5$ & $0.6148(4)$ & $-0.2039(4)$ & $1.0014(2)$ & $0.0693(10)$ \\
\hline H5 & 0.616239 & -0.242175 & 1.058118 & $0.083 *$ \\
\hline C6 & $0.6809(4)$ & $-0.1287(4)$ & $0.9749(2)$ & $0.0681(10)$ \\
\hline H6A & 0.728290 & -0.115280 & 1.013370 & $0.082 *$ \\
\hline $\mathrm{C} 7$ & $0.6789(4)$ & $-0.0715(3)$ & $0.8912(2)$ & $0.0563(8)$ \\
\hline $\mathrm{H} 7$ & 0.724585 & -0.019298 & 0.873923 & $0.068^{*}$ \\
\hline $\mathrm{C} 8$ & $0.3369(2)$ & $0.4028(3)$ & $0.71862(16)$ & $0.0310(5)$ \\
\hline C9 & $0.2075(3)$ & $0.4315(3)$ & $0.78336(17)$ & $0.0409(7)$ \\
\hline $\mathrm{C} 10$ & $0.1151(3)$ & $0.5588(4)$ & $0.8150(2)$ & $0.0631(9)$ \\
\hline $\mathrm{H} 10$ & 0.133562 & 0.630937 & 0.796580 & $0.076^{*}$ \\
\hline C11 & $-0.0048(4)$ & $0.5792(6)$ & $0.8740(3)$ & $0.0937(15)$ \\
\hline H11 & -0.066925 & 0.665074 & 0.895052 & $0.112^{*}$ \\
\hline $\mathrm{C} 12$ & $-0.0318(5)$ & $0.4751(7)$ & 0.9009 (3) & $0.0989(17)$ \\
\hline H12 & -0.112158 & 0.489572 & 0.941059 & $0.119^{*}$ \\
\hline $\mathrm{C} 13$ & $0.0568(4)$ & $0.3492(6)$ & $0.8701(3)$ & $0.0827(13)$ \\
\hline $\mathrm{H} 13$ & 0.036416 & 0.278359 & 0.888977 & $0.099^{*}$ \\
\hline $\mathrm{C} 14$ & $0.1782(3)$ & $0.3254(4)$ & $0.8102(2)$ & $0.0588(9)$ \\
\hline $\mathrm{H} 14$ & 0.238632 & 0.239352 & 0.788698 & $0.071^{*}$ \\
\hline $\mathrm{C} 15$ & $0.7799(3)$ & $0.2250(2)$ & $0.77007(17)$ & $0.0335(6)$ \\
\hline $\mathrm{C} 16$ & $0.7760(3)$ & $0.2465(3)$ & $0.86489(18)$ & $0.0394(6)$ \\
\hline $\mathrm{C} 17$ & $0.8465(4)$ & $0.1409(4)$ & $0.9196(2)$ & $0.0587(9)$ \\
\hline H17 & 0.901844 & 0.054955 & 0.896659 & $0.070^{*}$ \\
\hline $\mathrm{C} 18$ & $0.8347(4)$ & $0.1631(5)$ & $1.0086(3)$ & $0.0787(12)$ \\
\hline H18 & 0.881011 & 0.091693 & 1.045469 & $0.094 *$ \\
\hline C19 & $0.7552(4)$ & $0.2898(5)$ & $1.0424(3)$ & $0.0776(12)$ \\
\hline H19 & 0.748237 & 0.304574 & 1.102061 & $0.093^{*}$ \\
\hline $\mathrm{C} 20$ & $0.6865(4)$ & $0.3939(4)$ & $0.9889(2)$ & $0.0665(10)$ \\
\hline $\mathrm{H} 20$ & 0.633608 & 0.480011 & 1.012134 & $0.080^{*}$ \\
\hline $\mathrm{C} 21$ & $0.6941(3)$ & $0.3734(3)$ & $0.9001(2)$ & $0.0529(8)$ \\
\hline $\mathrm{H} 21$ & 0.644298 & 0.444845 & 0.864386 & $0.064^{*}$ \\
\hline $\mathrm{C} 22$ & $0.8656(2)$ & $0.2830(2)$ & $0.45328(16)$ & $0.0280(5)$ \\
\hline $\mathrm{C} 23$ & $0.9215(2)$ & $0.3801(2)$ & $0.42054(15)$ & $0.0243(5)$ \\
\hline $\mathrm{C} 24$ & $0.8477(2)$ & $0.5137(2)$ & $0.44522(15)$ & $0.0233(5)$ \\
\hline $\mathrm{H} 24$ & 0.761853 & 0.543431 & 0.482257 & $0.028^{*}$ \\
\hline $\mathrm{C} 25$ & $0.9000(2)$ & $0.6041(2)$ & $0.41547(15)$ & $0.0239(5)$ \\
\hline $\mathrm{C} 26$ & $0.8210(2)$ & $0.7487(2)$ & $0.44296(16)$ & $0.0275(5)$ \\
\hline $\mathrm{C} 27$ & $1.0285(3)$ & $0.5579(2)$ & $0.36103(17)$ & $0.0336(6)$ \\
\hline $\mathrm{H} 27$ & 1.064915 & 0.617294 & 0.341062 & $0.040^{*}$ \\
\hline $\mathrm{C} 28$ & $1.1032(3)$ & $0.4249(3)$ & $0.33605(19)$ & $0.0398(6)$ \\
\hline $\mathrm{H} 28$ & 1.189294 & 0.395113 & 0.299318 & $0.048^{*}$ \\
\hline $\mathrm{C} 29$ & $1.0498(2)$ & $0.3361(2)$ & $0.36574(17)$ & $0.0331(6)$ \\
\hline H29 & 1.100071 & 0.246465 & 0.348907 & $0.040 *$ \\
\hline
\end{tabular}


Atomic displacement parameters $\left(\AA^{2}\right)$

\begin{tabular}{|c|c|c|c|c|c|c|}
\hline & $U^{11}$ & $U^{22}$ & $U^{33}$ & $U^{12}$ & $U^{13}$ & $U^{23}$ \\
\hline Sm1 & $0.02326(7)$ & $0.01824(6)$ & $0.03323(7)$ & $-0.01103(5)$ & $-0.00329(5)$ & $0.00044(4)$ \\
\hline $\mathrm{O} 1$ & $0.0417(10)$ & $0.0396(10)$ & $0.0507(11)$ & $-0.0292(9)$ & $-0.0171(9)$ & $0.0155(9)$ \\
\hline $\mathrm{O} 2$ & $0.0333(10)$ & $0.0308(10)$ & $0.0500(11)$ & $-0.0170(8)$ & $0.0014(8)$ & $-0.0011(8)$ \\
\hline $\mathrm{O} 3$ & $0.0314(10)$ & $0.0306(9)$ & $0.0443(11)$ & $-0.0085(8)$ & $-0.0096(8)$ & $-0.0066(8)$ \\
\hline $\mathrm{O} 4$ & $0.0293(9)$ & $0.0208(8)$ & $0.0441(10)$ & $-0.0136(7)$ & $0.0044(8)$ & $-0.0035(7)$ \\
\hline $\mathrm{O} 5$ & $0.0391(11)$ & $0.0220(9)$ & $0.0847(15)$ & $-0.0165(8)$ & $0.0179(10)$ & $-0.0171(9)$ \\
\hline O6 & $0.0241(9)$ & $0.0201(8)$ & $0.0562(11)$ & $-0.0096(7)$ & $-0.0036(8)$ & $-0.0069(8)$ \\
\hline $\mathrm{O} 7$ & $0.0390(10)$ & $0.0245(9)$ & $0.0792(14)$ & $-0.0221(8)$ & $0.0088(10)$ & $-0.0119(9)$ \\
\hline O8 & $0.0371(10)$ & $0.0243(10)$ & $0.0609(13)$ & $-0.0182(9)$ & $0.0106(9)$ & $-0.0149(9)$ \\
\hline O9 & $0.0272(10)$ & $0.0291(9)$ & $0.0395(11)$ & $-0.0144(8)$ & $-0.0041(8)$ & $-0.0002(8)$ \\
\hline O10A & $0.034(5)$ & 0.093 & $0.072(7)$ & $-0.031(6)$ & $-0.010(4)$ & $0.008(7)$ \\
\hline O10B & $0.058(3)$ & $0.078(4)$ & $0.100(4)$ & $-0.048(3)$ & $0.002(3)$ & $-0.017(3)$ \\
\hline O11A & $0.071(10)$ & $0.050(6)$ & $0.152(12)$ & $-0.030(7)$ & $-0.025(9)$ & $-0.011(6)$ \\
\hline O11B & $0.079(5)$ & $0.052(3)$ & $0.103(4)$ & $-0.020(3)$ & $-0.028(4)$ & $-0.016(2)$ \\
\hline $\mathrm{O} 12 \mathrm{~A}$ & $0.058(8)$ & $0.051(7)$ & $0.078(7)$ & $0.014(5)$ & $-0.016(6)$ & $-0.020(6)$ \\
\hline O12B & $0.047(2)$ & $0.084(4)$ & $0.058(3)$ & $-0.034(2)$ & $-0.0040(18)$ & -0.014 \\
\hline N1 & $0.0333(12)$ & 0.0280 & $0.0354(12)$ & $-0.0163(10)$ & $-0.0081(10)$ & $0.0026(9)$ \\
\hline N2 & $0.0415(13)$ & $0.0336(12)$ & $0.0398(12)$ & $-0.0271(11)$ & $-0.0086(10)$ & $0.0078(10)$ \\
\hline N3 & $0.0352(13)$ & $0.0315(12)$ & $0.0379(13)$ & $-0.0162(10)$ & $0.0008(10)$ & $0.0016(10)$ \\
\hline N4 & $0.0304(11)$ & $0.0271(11)$ & $0.0343(12)$ & $-0.0093(10)$ & $0.0023(9)$ & $-0.0047(9)$ \\
\hline N5 & $0.0268(11)$ & $0.0254(11)$ & $0.0417(13)$ & $-0.0120(9)$ & $-0.0010(10)$ & $-0.0031(10)$ \\
\hline N6 & $0.0268(11)$ & $0.0359(12)$ & $0.0439(13)$ & $-0.0126(10)$ & $-0.0095(10)$ & $-0.0014(10)$ \\
\hline N7A & $0.018(5)$ & $0.027(6)$ & $0.038(9)$ & $0.000(4)$ & $-0.017(5)$ & $0.018(5)$ \\
\hline N7B & $0.066(5)$ & $0.072(5)$ & $0.046(5)$ & $-0.044(4)$ & $-0.007(4)$ & $-0.005(4)$ \\
\hline $\mathrm{C} 1$ & $0.0306(13)$ & $0.0241(12)$ & $0.0408(15)$ & $-0.0122(11)$ & $-0.0054(11)$ & $0.0041(11)$ \\
\hline $\mathrm{C} 2$ & $0.0374(14)$ & $0.0297(13)$ & $0.0412(15)$ & $-0.0150(12)$ & $-0.0072(12)$ & $0.0067(11)$ \\
\hline $\mathrm{C} 3$ & $0.088(3)$ & $0.081(3)$ & $0.064(2)$ & $-0.065(2)$ & $-0.033(2)$ & 0.0345 (19) \\
\hline $\mathrm{C} 4$ & $0.104(3)$ & $0.103(3)$ & $0.078(3)$ & $-0.077(3)$ & $-0.032(3)$ & $0.050(2)$ \\
\hline $\mathrm{C} 5$ & $0.078(3)$ & $0.071(2)$ & $0.050(2)$ & $-0.030(2)$ & $-0.0131(19)$ & $0.0227(18)$ \\
\hline C6 & $0.086(3)$ & $0.074(2)$ & $0.051(2)$ & -0.040 & $-0.0280(19)$ & $0.0132(18)$ \\
\hline $\mathrm{C} 7$ & $0.072(2)$ & $0.061(2)$ & 0.0528 (19) & $-0.0429(19)$ & $-0.0210(17)$ & $0.0143(16)$ \\
\hline $\mathrm{C} 8$ & $0.0278(13)$ & $0.0377(14)$ & $0.0286(13)$ & $-0.0150(11)$ & $-0.0084(10)$ & $0.0012(11)$ \\
\hline C9 & $0.0301(14)$ & 0.0648 (19) & $0.0301(14)$ & $-0.0244(14)$ & $-0.0032(11)$ & $-0.0007(13)$ \\
\hline $\mathrm{C} 10$ & $0.0447(18)$ & $0.083(3)$ & $0.051(2)$ & $-0.0249(18)$ & $0.0114(15)$ & $-0.0221(18)$ \\
\hline C11 & $0.054(2)$ & $0.134(4)$ & $0.074(3)$ & $-0.035(3)$ & $0.026(2)$ & -0.038 \\
\hline $\mathrm{C} 12$ & $0.060(3)$ & $0.179(6)$ & $0.059(3)$ & $-0.064(3)$ & $0.017(2)$ & $-0.014(3)$ \\
\hline C13 & $0.075(3)$ & $0.144(4)$ & $0.059(2)$ & -0.080 & $-0.007(2)$ & $0.021(3)$ \\
\hline $\mathrm{C} 14$ & $0.0502(19)$ & $0.087(3)$ & $0.0506(19)$ & $-0.0439(19)$ & $-0.0074(15)$ & $0.0128(18)$ \\
\hline $\mathrm{C} 15$ & $0.0331(14)$ & $0.0297(13)$ & $0.0428(15)$ & $-0.0184(12)$ & $-0.0061(12)$ & $-0.0020(11)$ \\
\hline $\mathrm{C} 16$ & $0.0355(14)$ & $0.0465(16)$ & $0.0438(16)$ & $-0.0234(13)$ & $-0.0098(12)$ & $-0.0026(13)$ \\
\hline $\mathrm{C} 17$ & $0.057(2)$ & $0.064(2)$ & $0.055(2)$ & $-0.0227(17)$ & $-0.0228(17)$ & $-0.0007(17)$ \\
\hline C18 & $0.078(3)$ & 0.099 (3) & 0.059 (2) & $-0.033(2)$ & $-0.035(2)$ & $0.008(2)$ \\
\hline C19 & $0.076(3)$ & $0.120(4)$ & $0.051(2)$ & $-0.053(3)$ & $-0.017(2)$ & $-0.012(2)$ \\
\hline $\mathrm{C} 20$ & $0.073(2)$ & $0.078(3)$ & $0.056(2)$ & $-0.041(2)$ & $0.0006(19)$ & $-0.0246(19)$ \\
\hline $\mathrm{C} 21$ & $0.0538(19)$ & $0.0540(19)$ & $0.0530(19)$ & $-0.0261(16)$ & $-0.0056(15)$ & $-0.0091(15)$ \\
\hline
\end{tabular}




\begin{tabular}{lllllll}
\hline & & & & & \\
$\mathrm{C} 22$ & $0.0292(13)$ & $0.0189(11)$ & $0.0365(14)$ & $-0.0116(10)$ & $-0.0051(11)$ & $-0.0014(10)$ \\
$\mathrm{C} 23$ & $0.0227(11)$ & $0.0205(11)$ & $0.0303(12)$ & $-0.0105(9)$ & $-0.0044(10)$ & $0.0005(9)$ \\
$\mathrm{C} 24$ & $0.0215(11)$ & $0.0209(11)$ & $0.0283(12)$ & $-0.0106(9)$ & $-0.0039(9)$ & $-0.0006(9)$ \\
C25 & $0.0238(11)$ & $0.0197(11)$ & $0.0314(12)$ & $-0.0117(9)$ & $-0.0075(10)$ & $0.0002(9)$ \\
C26 & $0.0268(12)$ & $0.0204(11)$ & $0.0382(14)$ & $-0.0123(10)$ & $-0.0081(11)$ & $-0.0005(10)$ \\
C27 & $0.0320(13)$ & $0.0282(13)$ & $0.0448(15)$ & $-0.0201(11)$ & $0.0016(11)$ & $-0.0016(11)$ \\
C28 & $0.0286(13)$ & $0.0324(14)$ & $0.0534(17)$ & $-0.0154(11)$ & $0.0117(12)$ & $-0.0102(12)$ \\
C29 & $0.0290(13)$ & $0.0210(12)$ & $0.0448(15)$ & $-0.0099(10)$ & $0.0032(11)$ & $-0.0087(11)$ \\
\hline
\end{tabular}

Geometric parameters $\left(\AA,{ }^{\circ}\right)$

\begin{tabular}{|c|c|c|c|}
\hline $\mathrm{Sm} 1-\mathrm{O} 1$ & $2.4415(17)$ & $\mathrm{C} 3-\mathrm{C} 4$ & $1.385(5)$ \\
\hline $\mathrm{Sm} 1-\mathrm{O} 2$ & $2.3973(17)$ & $\mathrm{C} 4-\mathrm{H} 4 \mathrm{~A}$ & 0.9300 \\
\hline $\mathrm{Sm} 1-\mathrm{O} 3$ & $2.4775(17)$ & $\mathrm{C} 4-\mathrm{C} 5$ & $1.348(5)$ \\
\hline $\mathrm{Sm} 1-\mathrm{O} 4$ & $2.4024(16)$ & $\mathrm{C} 5-\mathrm{H} 5$ & 0.9300 \\
\hline $\mathrm{Sm} 1-\mathrm{O} 8$ & $2.3397(19)$ & $\mathrm{C} 5-\mathrm{C} 6$ & $1.344(5)$ \\
\hline $\mathrm{Sm} 1-\mathrm{O} 9$ & $2.4827(18)$ & C6-H6A & 0.9300 \\
\hline $\mathrm{Sm} 1-\mathrm{N} 1$ & $2.694(2)$ & $\mathrm{C} 6-\mathrm{C} 7$ & $1.375(4)$ \\
\hline $\mathrm{Sm} 1-\mathrm{N} 3$ & $2.680(2)$ & $\mathrm{C} 7-\mathrm{H} 7$ & 0.9300 \\
\hline $\mathrm{Sm} 1-\mathrm{N} 5$ & $2.633(2)$ & $\mathrm{C} 8-\mathrm{C} 9$ & $1.487(3)$ \\
\hline $\mathrm{O} 1-\mathrm{C} 1$ & $1.243(3)$ & $\mathrm{C} 9-\mathrm{C} 10$ & $1.381(4)$ \\
\hline $\mathrm{O} 2-\mathrm{C} 8$ & $1.240(3)$ & $\mathrm{C} 9-\mathrm{C} 14$ & $1.380(4)$ \\
\hline $\mathrm{O} 3-\mathrm{C} 15$ & $1.246(3)$ & $\mathrm{C} 10-\mathrm{H} 10$ & 0.9300 \\
\hline $\mathrm{O} 4-\mathrm{C} 22$ & $1.277(3)$ & $\mathrm{C} 10-\mathrm{C} 11$ & $1.384(5)$ \\
\hline $\mathrm{O} 5-\mathrm{C} 22$ & $1.238(3)$ & C11-H11 & 0.9300 \\
\hline $\mathrm{O} 6-\mathrm{C} 26$ & $1.262(3)$ & $\mathrm{C} 11-\mathrm{C} 12$ & $1.346(7)$ \\
\hline $\mathrm{O} 7-\mathrm{C} 26$ & $1.244(3)$ & $\mathrm{C} 12-\mathrm{H} 12$ & 0.9300 \\
\hline $\mathrm{O} 8-\mathrm{H} 8 \mathrm{~A}$ & $0.834(10)$ & $\mathrm{C} 12-\mathrm{C} 13$ & $1.358(7)$ \\
\hline $\mathrm{O} 8-\mathrm{H} 8 \mathrm{~B}$ & $0.837(10)$ & $\mathrm{C} 13-\mathrm{H} 13$ & 0.9300 \\
\hline O9- $\mathrm{H} 9 \mathrm{~A}$ & $0.825(10)$ & $\mathrm{C} 13-\mathrm{C} 14$ & $1.395(5)$ \\
\hline O9-H9B & $0.831(10)$ & $\mathrm{C} 14-\mathrm{H} 14$ & 0.9300 \\
\hline $\mathrm{O} 10 \mathrm{~A}-\mathrm{N} 7 \mathrm{~A}$ & $1.238(12)$ & $\mathrm{C} 15-\mathrm{C} 16$ & $1.490(4)$ \\
\hline O10B-N7B & $1.234(8)$ & $\mathrm{C} 16-\mathrm{C} 17$ & $1.383(4)$ \\
\hline O11A $-\mathrm{N} 7 \mathrm{~A}$ & $1.242(13)$ & $\mathrm{C} 16-\mathrm{C} 21$ & $1.380(4)$ \\
\hline $\mathrm{O} 11 \mathrm{~B}-\mathrm{N} 7 \mathrm{~B}$ & $1.235(8)$ & $\mathrm{C} 17-\mathrm{H} 17$ & 0.9300 \\
\hline $\mathrm{O} 12 \mathrm{~A}-\mathrm{N} 7 \mathrm{~A}$ & $1.257(12)$ & $\mathrm{C} 17-\mathrm{C} 18$ & $1.384(5)$ \\
\hline $\mathrm{O} 12 \mathrm{~B}-\mathrm{N} 7 \mathrm{~B}$ & $1.238(8)$ & $\mathrm{C} 18-\mathrm{H} 18$ & 0.9300 \\
\hline $\mathrm{N} 1-\mathrm{H} 1 \mathrm{~A}$ & $0.875(10)$ & $\mathrm{C} 18-\mathrm{C} 19$ & $1.368(6)$ \\
\hline $\mathrm{N} 1-\mathrm{H} 1 \mathrm{~B}$ & $0.878(10)$ & $\mathrm{C} 19-\mathrm{H} 19$ & 0.9300 \\
\hline $\mathrm{N} 1-\mathrm{N} 2$ & $1.413(3)$ & $\mathrm{C} 19-\mathrm{C} 20$ & $1.356(5)$ \\
\hline $\mathrm{N} 2-\mathrm{H} 2$ & $0.871(10)$ & $\mathrm{C} 20-\mathrm{H} 20$ & 0.9300 \\
\hline $\mathrm{N} 2-\mathrm{C} 1$ & $1.320(3)$ & $\mathrm{C} 20-\mathrm{C} 21$ & $1.387(5)$ \\
\hline $\mathrm{N} 3-\mathrm{H} 3 \mathrm{~A}$ & $0.870(10)$ & $\mathrm{C} 21-\mathrm{H} 21$ & 0.9300 \\
\hline N3-H3B & $0.878(10)$ & $\mathrm{C} 22-\mathrm{C} 23$ & $1.492(3)$ \\
\hline $\mathrm{N} 3-\mathrm{N} 4$ & $1.410(3)$ & $\mathrm{C} 23-\mathrm{C} 24$ & $1.384(3)$ \\
\hline N4-H4 & $0.867(10)$ & $\mathrm{C} 23-\mathrm{C} 29$ & $1.387(3)$ \\
\hline $\mathrm{N} 4-\mathrm{C} 8$ & $1.322(3)$ & $\mathrm{C} 24-\mathrm{H} 24$ & 0.9300 \\
\hline N5-H5A & $0.879(10)$ & $\mathrm{C} 24-\mathrm{C} 25$ & $1.388(3)$ \\
\hline
\end{tabular}




\begin{tabular}{|c|c|c|c|}
\hline N5-H5B & $0.871(10)$ & $\mathrm{C} 25-\mathrm{C} 26$ & $1.501(3)$ \\
\hline $\mathrm{N} 5-\mathrm{N} 6$ & $1.410(3)$ & $\mathrm{C} 25-\mathrm{C} 27$ & $1.385(3)$ \\
\hline N6-H6 & $0.873(10)$ & $\mathrm{C} 27-\mathrm{H} 27$ & 0.9300 \\
\hline N6-C15 & $1.319(3)$ & $\mathrm{C} 27-\mathrm{C} 28$ & $1.379(3)$ \\
\hline $\mathrm{C} 1-\mathrm{C} 2$ & $1.489(3)$ & $\mathrm{C} 28-\mathrm{H} 28$ & 0.9300 \\
\hline $\mathrm{C} 2-\mathrm{C} 3$ & $1.373(4)$ & $\mathrm{C} 28-\mathrm{C} 29$ & $1.381(3)$ \\
\hline $\mathrm{C} 2-\mathrm{C} 7$ & $1.370(4)$ & $\mathrm{C} 29-\mathrm{H} 29$ & 0.9300 \\
\hline $\mathrm{C} 3-\mathrm{H} 3$ & 0.9300 & & \\
\hline $\mathrm{O} 1-\mathrm{Sm} 1-\mathrm{O} 3$ & $72.08(6)$ & $\mathrm{C} 7-\mathrm{C} 2-\mathrm{C} 1$ & $117.9(2)$ \\
\hline $\mathrm{O} 1-\mathrm{Sm} 1-\mathrm{O} 9$ & $133.85(6)$ & $\mathrm{C} 7-\mathrm{C} 2-\mathrm{C} 3$ & $118.2(3)$ \\
\hline $\mathrm{O} 1-\mathrm{Sm} 1-\mathrm{N} 1$ & $60.97(6)$ & $\mathrm{C} 2-\mathrm{C} 3-\mathrm{H} 3$ & 120.1 \\
\hline $\mathrm{O} 1-\mathrm{Sm} 1-\mathrm{N} 3$ & $125.95(7)$ & $\mathrm{C} 2-\mathrm{C} 3-\mathrm{C} 4$ & $119.8(3)$ \\
\hline $\mathrm{O} 1-\mathrm{Sm} 1-\mathrm{N} 5$ & $67.85(6)$ & $\mathrm{C} 4-\mathrm{C} 3-\mathrm{H} 3$ & 120.1 \\
\hline $\mathrm{O} 2-\mathrm{Sm} 1-\mathrm{O} 1$ & $77.69(6)$ & $\mathrm{C} 3-\mathrm{C} 4-\mathrm{H} 4 \mathrm{~A}$ & 119.5 \\
\hline $\mathrm{O} 2-\mathrm{Sm} 1-\mathrm{O} 3$ & $79.96(6)$ & $\mathrm{C} 5-\mathrm{C} 4-\mathrm{C} 3$ & $121.0(3)$ \\
\hline $\mathrm{O} 2-\mathrm{Sm} 1-\mathrm{O} 4$ & $131.15(6)$ & $\mathrm{C} 5-\mathrm{C} 4-\mathrm{H} 4 \mathrm{~A}$ & 119.5 \\
\hline $\mathrm{O} 2-\mathrm{Sm} 1-\mathrm{O} 9$ & $80.28(6)$ & $\mathrm{C} 4-\mathrm{C} 5-\mathrm{H} 5$ & 120.2 \\
\hline $\mathrm{O} 2-\mathrm{Sm} 1-\mathrm{N} 1$ & $68.37(6)$ & $\mathrm{C} 6-\mathrm{C} 5-\mathrm{C} 4$ & $119.6(3)$ \\
\hline $\mathrm{O} 2-\mathrm{Sm} 1-\mathrm{N} 3$ & $61.71(6)$ & $\mathrm{C} 6-\mathrm{C} 5-\mathrm{H} 5$ & 120.2 \\
\hline $\mathrm{O} 2-\mathrm{Sm} 1-\mathrm{N} 5$ & $134.49(7)$ & $\mathrm{C} 5-\mathrm{C} 6-\mathrm{H} 6 \mathrm{~A}$ & 119.8 \\
\hline $\mathrm{O} 3-\mathrm{Sm} 1-\mathrm{O} 9$ & $141.80(6)$ & $\mathrm{C} 5-\mathrm{C} 6-\mathrm{C} 7$ & $120.5(3)$ \\
\hline $\mathrm{O} 3-\mathrm{Sm} 1-\mathrm{N} 1$ & $127.30(6)$ & $\mathrm{C} 7-\mathrm{C} 6-\mathrm{H} 6 \mathrm{~A}$ & 119.8 \\
\hline $\mathrm{O} 3-\mathrm{Sm} 1-\mathrm{N} 3$ & $67.24(7)$ & $\mathrm{C} 2-\mathrm{C} 7-\mathrm{C} 6$ & $120.9(3)$ \\
\hline $\mathrm{O} 3-\mathrm{Sm} 1-\mathrm{N} 5$ & $62.03(6)$ & $\mathrm{C} 2-\mathrm{C} 7-\mathrm{H} 7$ & 119.5 \\
\hline $\mathrm{O} 4-\mathrm{Sm} 1-\mathrm{O} 1$ & $144.08(6)$ & $\mathrm{C} 6-\mathrm{C} 7-\mathrm{H} 7$ & 119.5 \\
\hline $\mathrm{O} 4-\mathrm{Sm} 1-\mathrm{O} 3$ & $90.56(6)$ & $\mathrm{O} 2-\mathrm{C} 8-\mathrm{N} 4$ & $121.2(2)$ \\
\hline $\mathrm{O} 4-\mathrm{Sm} 1-\mathrm{O} 9$ & $78.51(6)$ & $\mathrm{O} 2-\mathrm{C} 8-\mathrm{C} 9$ & $120.1(2)$ \\
\hline $\mathrm{O} 4-\mathrm{Sm} 1-\mathrm{N} 1$ & $141.91(6)$ & $\mathrm{N} 4-\mathrm{C} 8-\mathrm{C} 9$ & $118.7(2)$ \\
\hline $\mathrm{O} 4-\mathrm{Sm} 1-\mathrm{N} 3$ & $70.39(6)$ & $\mathrm{C} 10-\mathrm{C} 9-\mathrm{C} 8$ & $122.9(3)$ \\
\hline $\mathrm{O} 4-\mathrm{Sm} 1-\mathrm{N} 5$ & $76.25(6)$ & $\mathrm{C} 14-\mathrm{C} 9-\mathrm{C} 8$ & $117.6(3)$ \\
\hline $\mathrm{O} 8-\mathrm{Sm} 1-\mathrm{O} 1$ & $95.65(7)$ & $\mathrm{C} 14-\mathrm{C} 9-\mathrm{C} 10$ & $119.5(3)$ \\
\hline $\mathrm{O} 8-\mathrm{Sm} 1-\mathrm{O} 2$ & $137.16(6)$ & $\mathrm{C} 9-\mathrm{C} 10-\mathrm{H} 10$ & 120.0 \\
\hline $\mathrm{O} 8-\mathrm{Sm} 1-\mathrm{O} 3$ & $138.72(7)$ & $\mathrm{C} 9-\mathrm{C} 10-\mathrm{C} 11$ & $120.1(4)$ \\
\hline $\mathrm{O} 8-\mathrm{Sm} 1-\mathrm{O} 4$ & $76.66(6)$ & $\mathrm{C} 11-\mathrm{C} 10-\mathrm{H} 10$ & 120.0 \\
\hline $\mathrm{O} 8-\mathrm{Sm} 1-\mathrm{O} 9$ & $74.43(7)$ & $\mathrm{C} 10-\mathrm{C} 11-\mathrm{H} 11$ & 119.9 \\
\hline $\mathrm{O} 8-\mathrm{Sm} 1-\mathrm{N} 1$ & $71.49(7)$ & $\mathrm{C} 12-\mathrm{C} 11-\mathrm{C} 10$ & $120.2(4)$ \\
\hline $\mathrm{O} 8-\mathrm{Sm} 1-\mathrm{N} 3$ & $138.35(7)$ & $\mathrm{C} 12-\mathrm{C} 11-\mathrm{H} 11$ & 119.9 \\
\hline $\mathrm{O} 8-\mathrm{Sm} 1-\mathrm{N} 5$ & $76.77(7)$ & $\mathrm{C} 11-\mathrm{C} 12-\mathrm{H} 12$ & 119.6 \\
\hline $\mathrm{O} 9-\mathrm{Sm} 1-\mathrm{N} 1$ & $73.31(6)$ & $\mathrm{C} 11-\mathrm{C} 12-\mathrm{C} 13$ & $120.8(4)$ \\
\hline $\mathrm{O} 9-\mathrm{Sm} 1-\mathrm{N} 3$ & $74.63(7)$ & $\mathrm{C} 13-\mathrm{C} 12-\mathrm{H} 12$ & 119.6 \\
\hline $\mathrm{O} 9-\mathrm{Sm} 1-\mathrm{N} 5$ & $145.24(6)$ & $\mathrm{C} 12-\mathrm{C} 13-\mathrm{H} 13$ & 119.8 \\
\hline $\mathrm{N} 3-\mathrm{Sm} 1-\mathrm{N} 1$ & $123.90(7)$ & $\mathrm{C} 12-\mathrm{C} 13-\mathrm{C} 14$ & $120.4(4)$ \\
\hline $\mathrm{N} 5-\mathrm{Sm} 1-\mathrm{N} 1$ & $115.01(7)$ & $\mathrm{C} 14-\mathrm{C} 13-\mathrm{H} 13$ & 119.8 \\
\hline $\mathrm{N} 5-\mathrm{Sm} 1-\mathrm{N} 3$ & $117.67(7)$ & $\mathrm{C} 9-\mathrm{C} 14-\mathrm{C} 13$ & $119.0(4)$ \\
\hline $\mathrm{C} 1-\mathrm{O} 1-\mathrm{Sm} 1$ & $124.68(16)$ & C9-C14-H14 & 120.5 \\
\hline $\mathrm{C} 8-\mathrm{O} 2-\mathrm{Sm} 1$ & $126.68(16)$ & $\mathrm{C} 13-\mathrm{C} 14-\mathrm{H} 14$ & 120.5 \\
\hline $\mathrm{C} 15-\mathrm{O} 3-\mathrm{Sm} 1$ & $120.32(16)$ & $\mathrm{O} 3-\mathrm{C} 15-\mathrm{N} 6$ & $121.8(2)$ \\
\hline
\end{tabular}




\begin{tabular}{|c|c|c|c|}
\hline $\mathrm{C} 22-\mathrm{O} 4-\mathrm{Sm} 1$ & $130.94(14)$ & $\mathrm{O} 3-\mathrm{C} 15-\mathrm{C} 16$ & $120.6(2)$ \\
\hline $\mathrm{Sm} 1-\mathrm{O} 8-\mathrm{H} 8 \mathrm{~A}$ & $144(2)$ & $\mathrm{N} 6-\mathrm{C} 15-\mathrm{C} 16$ & $117.5(2)$ \\
\hline $\mathrm{Sm} 1-\mathrm{O} 8-\mathrm{H} 8 \mathrm{~B}$ & $104(3)$ & $\mathrm{C} 17-\mathrm{C} 16-\mathrm{C} 15$ & $121.6(3)$ \\
\hline $\mathrm{H} 8 \mathrm{~A}-\mathrm{O} 8-\mathrm{H} 8 \mathrm{~B}$ & $109(3)$ & $\mathrm{C} 21-\mathrm{C} 16-\mathrm{C} 15$ & $119.1(3)$ \\
\hline $\mathrm{Sm} 1-\mathrm{O} 9-\mathrm{H} 9 \mathrm{~A}$ & $110.4(19)$ & $\mathrm{C} 21-\mathrm{C} 16-\mathrm{C} 17$ & $119.2(3)$ \\
\hline $\mathrm{Sm} 1-\mathrm{O} 9-\mathrm{H} 9 \mathrm{~B}$ & $117(2)$ & $\mathrm{C} 16-\mathrm{C} 17-\mathrm{H} 17$ & 120.0 \\
\hline $\mathrm{H} 9 \mathrm{~A}-\mathrm{O} 9-\mathrm{H} 9 \mathrm{~B}$ & $106(3)$ & $\mathrm{C} 16-\mathrm{C} 17-\mathrm{C} 18$ & $120.1(3)$ \\
\hline $\mathrm{Sm} 1-\mathrm{N} 1-\mathrm{H} 1 \mathrm{~A}$ & $114.3(18)$ & $\mathrm{C} 18-\mathrm{C} 17-\mathrm{H} 17$ & 120.0 \\
\hline $\mathrm{Sm} 1-\mathrm{N} 1-\mathrm{H} 1 \mathrm{~B}$ & $109.2(19)$ & $\mathrm{C} 17-\mathrm{C} 18-\mathrm{H} 18$ & 119.9 \\
\hline $\mathrm{H} 1 \mathrm{~A}-\mathrm{N} 1-\mathrm{H} 1 \mathrm{~B}$ & $105(3)$ & $\mathrm{C} 19-\mathrm{C} 18-\mathrm{C} 17$ & $120.2(4)$ \\
\hline $\mathrm{N} 2-\mathrm{N} 1-\mathrm{Sm} 1$ & $111.02(14)$ & $\mathrm{C} 19-\mathrm{C} 18-\mathrm{H} 18$ & 119.9 \\
\hline $\mathrm{N} 2-\mathrm{N} 1-\mathrm{H} 1 \mathrm{~A}$ & $107.0(17)$ & $\mathrm{C} 18-\mathrm{C} 19-\mathrm{H} 19$ & 120.0 \\
\hline $\mathrm{N} 2-\mathrm{N} 1-\mathrm{H} 1 \mathrm{~B}$ & $110.1(19)$ & $\mathrm{C} 20-\mathrm{C} 19-\mathrm{C} 18$ & 120.0 \\
\hline $\mathrm{N} 1-\mathrm{N} 2-\mathrm{H} 2$ & $119.1(18)$ & $\mathrm{C} 20-\mathrm{C} 19-\mathrm{H} 19$ & 120.0 \\
\hline $\mathrm{C} 1-\mathrm{N} 2-\mathrm{N} 1$ & $117.4(2)$ & $\mathrm{C} 19-\mathrm{C} 20-\mathrm{H} 20$ & 119.6 \\
\hline $\mathrm{C} 1-\mathrm{N} 2-\mathrm{H} 2$ & $123.5(18)$ & $\mathrm{C} 19-\mathrm{C} 20-\mathrm{C} 21$ & $120.7(4)$ \\
\hline $\mathrm{Sm} 1-\mathrm{N} 3-\mathrm{H} 3 \mathrm{~A}$ & $106(2)$ & $\mathrm{C} 21-\mathrm{C} 20-\mathrm{H} 20$ & 119.6 \\
\hline $\mathrm{Sm} 1-\mathrm{N} 3-\mathrm{H} 3 \mathrm{~B}$ & $116(2)$ & $\mathrm{C} 16-\mathrm{C} 21-\mathrm{C} 20$ & $119.7(3)$ \\
\hline $\mathrm{H} 3 \mathrm{~A}-\mathrm{N} 3-\mathrm{H} 3 \mathrm{~B}$ & $113(3)$ & $\mathrm{C} 16-\mathrm{C} 21-\mathrm{H} 21$ & 120.1 \\
\hline $\mathrm{N} 4-\mathrm{N} 3-\mathrm{Sm} 1$ & $111.54(14)$ & $\mathrm{C} 20-\mathrm{C} 21-\mathrm{H} 21$ & 120.1 \\
\hline $\mathrm{N} 4-\mathrm{N} 3-\mathrm{H} 3 \mathrm{~A}$ & $107(2)$ & $\mathrm{O} 4-\mathrm{C} 22-\mathrm{C} 23$ & 118.50 \\
\hline $\mathrm{N} 4-\mathrm{N} 3-\mathrm{H} 3 \mathrm{~B}$ & $103(2)$ & $\mathrm{O} 5-\mathrm{C} 22-\mathrm{O} 4$ & $123.6(2)$ \\
\hline $\mathrm{N} 3-\mathrm{N} 4-\mathrm{H} 4$ & $116(2)$ & $\mathrm{O} 5-\mathrm{C} 22-\mathrm{C} 23$ & $117.9(2)$ \\
\hline $\mathrm{C} 8-\mathrm{N} 4-\mathrm{N} 3$ & $117.1(2)$ & $\mathrm{C} 24-\mathrm{C} 23-\mathrm{C} 22$ & $120.8(2)$ \\
\hline $\mathrm{C} 8-\mathrm{N} 4-\mathrm{H} 4$ & $126(2)$ & $\mathrm{C} 24-\mathrm{C} 23-\mathrm{C} 29$ & $119.3(2)$ \\
\hline $\mathrm{Sm} 1-\mathrm{N} 5-\mathrm{H} 5 \mathrm{~A}$ & $106.2(18)$ & $\mathrm{C} 29-\mathrm{C} 23-\mathrm{C} 22$ & $119.9(2)$ \\
\hline $\mathrm{Sm} 1-\mathrm{N} 5-\mathrm{H} 5 \mathrm{~B}$ & $113.6(18)$ & $\mathrm{C} 23-\mathrm{C} 24-\mathrm{H} 24$ & 119.5 \\
\hline $\mathrm{H} 5 \mathrm{~A}-\mathrm{N} 5-\mathrm{H} 5 \mathrm{~B}$ & $110(2)$ & $\mathrm{C} 23-\mathrm{C} 24-\mathrm{C} 25$ & $120.9(2)$ \\
\hline $\mathrm{N} 6-\mathrm{N} 5-\mathrm{Sm} 1$ & $111.50(14)$ & $\mathrm{C} 25-\mathrm{C} 24-\mathrm{H} 24$ & 119.5 \\
\hline $\mathrm{N} 6-\mathrm{N} 5-\mathrm{H} 5 \mathrm{~A}$ & $107.1(18)$ & $\mathrm{C} 24-\mathrm{C} 25-\mathrm{C} 26$ & $121.1(2)$ \\
\hline $\mathrm{N} 6-\mathrm{N} 5-\mathrm{H} 5 \mathrm{~B}$ & $108.7(18)$ & $\mathrm{C} 27-\mathrm{C} 25-\mathrm{C} 24$ & $118.8(2)$ \\
\hline $\mathrm{N} 5-\mathrm{N} 6-\mathrm{H} 6$ & $119.2(19)$ & $\mathrm{C} 27-\mathrm{C} 25-\mathrm{C} 26$ & $120.1(2)$ \\
\hline $\mathrm{C} 15-\mathrm{N} 6-\mathrm{N} 5$ & $117.6(2)$ & $\mathrm{O} 6-\mathrm{C} 26-\mathrm{C} 25$ & $119.4(2)$ \\
\hline $\mathrm{C} 15-\mathrm{N} 6-\mathrm{H} 6$ & $123.1(19)$ & $\mathrm{O} 7-\mathrm{C} 26-\mathrm{O} 6$ & $123.5(2)$ \\
\hline $\mathrm{O} 10 \mathrm{~A}-\mathrm{N} 7 \mathrm{~A}-\mathrm{O} 11 \mathrm{~A}$ & $116.6(12)$ & $\mathrm{O} 7-\mathrm{C} 26-\mathrm{C} 25$ & $117.1(2)$ \\
\hline $\mathrm{O} 10 \mathrm{~A}-\mathrm{N} 7 \mathrm{~A}-\mathrm{O} 12 \mathrm{~A}$ & $124.6(13)$ & $\mathrm{C} 25-\mathrm{C} 27-\mathrm{H} 27$ & 119.6 \\
\hline $\mathrm{O} 11 \mathrm{~A}-\mathrm{N} 7 \mathrm{~A}-\mathrm{O} 12 \mathrm{~A}$ & $118.5(13)$ & $\mathrm{C} 28-\mathrm{C} 27-\mathrm{C} 25$ & $120.9(2)$ \\
\hline $\mathrm{O} 10 \mathrm{~B}-\mathrm{N} 7 \mathrm{~B}-\mathrm{O} 11 \mathrm{~B}$ & $118.4(7)$ & $\mathrm{C} 28-\mathrm{C} 27-\mathrm{H} 27$ & 119.6 \\
\hline $\mathrm{O} 10 \mathrm{~B}-\mathrm{N} 7 \mathrm{~B}-\mathrm{O} 12 \mathrm{~B}$ & $119.9(7)$ & $\mathrm{C} 27-\mathrm{C} 28-\mathrm{H} 28$ & 120.1 \\
\hline $\mathrm{O} 11 \mathrm{~B}-\mathrm{N} 7 \mathrm{~B}-\mathrm{O} 12 \mathrm{~B}$ & $121.7(7)$ & $\mathrm{C} 27-\mathrm{C} 28-\mathrm{C} 29$ & $119.8(2)$ \\
\hline $\mathrm{O} 1-\mathrm{C} 1-\mathrm{N} 2$ & $120.8(2)$ & $\mathrm{C} 29-\mathrm{C} 28-\mathrm{H} 28$ & 120.1 \\
\hline $\mathrm{O} 1-\mathrm{C} 1-\mathrm{C} 2$ & $120.2(2)$ & $\mathrm{C} 23-\mathrm{C} 29-\mathrm{H} 29$ & 119.9 \\
\hline $\mathrm{N} 2-\mathrm{C} 1-\mathrm{C} 2$ & $119.0(2)$ & $\mathrm{C} 28-\mathrm{C} 29-\mathrm{C} 23$ & $120.3(2)$ \\
\hline $\mathrm{C} 3-\mathrm{C} 2-\mathrm{C} 1$ & $123.9(3)$ & $\mathrm{C} 28-\mathrm{C} 29-\mathrm{H} 29$ & 119.9 \\
\hline $\mathrm{Sm} 1-\mathrm{O} 1-\mathrm{C} 1-\mathrm{N} 2$ & $20.2(3)$ & $\mathrm{C} 3-\mathrm{C} 2-\mathrm{C} 7-\mathrm{C} 6$ & $0.4(5)$ \\
\hline $\mathrm{Sm} 1-\mathrm{O} 1-\mathrm{C} 1-\mathrm{C} 2$ & $-159.95(17)$ & $\mathrm{C} 3-\mathrm{C} 4-\mathrm{C} 5-\mathrm{C} 6$ & $0.0(7)$ \\
\hline $\mathrm{Sm} 1-\mathrm{O} 2-\mathrm{C} 8-\mathrm{N} 4$ & $8.8(3)$ & $\mathrm{C} 4-\mathrm{C} 5-\mathrm{C} 6-\mathrm{C} 7$ & $0.2(6)$ \\
\hline
\end{tabular}




\begin{tabular}{|c|c|}
\hline $\mathrm{Sm} 1-\mathrm{O} 2-\mathrm{C} 8-\mathrm{C} 9$ & $-171.58(16)$ \\
\hline $\mathrm{Sm} 1-\mathrm{O} 3-\mathrm{C} 15-\mathrm{N} 6$ & $24.0(3)$ \\
\hline $\mathrm{Sm} 1-\mathrm{O} 3-\mathrm{C} 15-\mathrm{C} 16$ & $-155.18(18)$ \\
\hline $\mathrm{Sm} 1-\mathrm{O} 4-\mathrm{C} 22-\mathrm{O} 5$ & $17.8(4)$ \\
\hline $\mathrm{Sm} 1-\mathrm{O} 4-\mathrm{C} 22-\mathrm{C} 23$ & $-161.23(15)$ \\
\hline $\mathrm{Sm} 1-\mathrm{N} 1-\mathrm{N} 2-\mathrm{C} 1$ & $-16.1(3)$ \\
\hline $\mathrm{Sm} 1-\mathrm{N} 3-\mathrm{N} 4-\mathrm{C} 8$ & $-12.0(3)$ \\
\hline Sm1-N5-N6-C15 & $-17.1(3)$ \\
\hline $\mathrm{O} 1-\mathrm{C} 1-\mathrm{C} 2-\mathrm{C} 3$ & $-176.1(3)$ \\
\hline $\mathrm{O} 1-\mathrm{C} 1-\mathrm{C} 2-\mathrm{C} 7$ & $5.1(4)$ \\
\hline $\mathrm{O} 2-\mathrm{C} 8-\mathrm{C} 9-\mathrm{C} 10$ & $-179.7(3)$ \\
\hline $\mathrm{O} 2-\mathrm{C} 8-\mathrm{C} 9-\mathrm{C} 14$ & $-1.7(4)$ \\
\hline $\mathrm{O} 3-\mathrm{C} 15-\mathrm{C} 16-\mathrm{C} 17$ & $146.7(3)$ \\
\hline $\mathrm{O} 3-\mathrm{C} 15-\mathrm{C} 16-\mathrm{C} 21$ & $-29.8(4)$ \\
\hline $\mathrm{O} 4-\mathrm{C} 22-\mathrm{C} 23-\mathrm{C} 24$ & $0.4(3)$ \\
\hline $\mathrm{O} 4-\mathrm{C} 22-\mathrm{C} 23-\mathrm{C} 29$ & $179.4(2)$ \\
\hline $\mathrm{O} 5-\mathrm{C} 22-\mathrm{C} 23-\mathrm{C} 24$ & $-178.7(2)$ \\
\hline $\mathrm{O} 5-\mathrm{C} 22-\mathrm{C} 23-\mathrm{C} 29$ & $0.4(4)$ \\
\hline $\mathrm{N} 1-\mathrm{N} 2-\mathrm{C} 1-\mathrm{O} 1$ & $0.1(4)$ \\
\hline $\mathrm{N} 1-\mathrm{N} 2-\mathrm{C} 1-\mathrm{C} 2$ & $-179.8(2)$ \\
\hline $\mathrm{N} 2-\mathrm{C} 1-\mathrm{C} 2-\mathrm{C} 3$ & $3.8(4)$ \\
\hline $\mathrm{N} 2-\mathrm{C} 1-\mathrm{C} 2-\mathrm{C} 7$ & $-175.0(3)$ \\
\hline $\mathrm{N} 3-\mathrm{N} 4-\mathrm{C} 8-\mathrm{O} 2$ & $4.0(4)$ \\
\hline $\mathrm{N} 3-\mathrm{N} 4-\mathrm{C} 8-\mathrm{C} 9$ & $-175.7(2)$ \\
\hline $\mathrm{N} 4-\mathrm{C} 8-\mathrm{C} 9-\mathrm{C} 10$ & $0.0(4)$ \\
\hline $\mathrm{N} 4-\mathrm{C} 8-\mathrm{C} 9-\mathrm{C} 14$ & $178.0(2)$ \\
\hline $\mathrm{N} 5-\mathrm{N} 6-\mathrm{C} 15-\mathrm{O} 3$ & $-2.7(4)$ \\
\hline $\mathrm{N} 5-\mathrm{N} 6-\mathrm{C} 15-\mathrm{C} 16$ & $176.5(2)$ \\
\hline $\mathrm{N} 6-\mathrm{C} 15-\mathrm{C} 16-\mathrm{C} 17$ & $-32.5(4)$ \\
\hline $\mathrm{N} 6-\mathrm{C} 15-\mathrm{C} 16-\mathrm{C} 21$ & $151.0(3)$ \\
\hline $\mathrm{C} 1-\mathrm{C} 2-\mathrm{C} 3-\mathrm{C} 4$ & $-179.0(3)$ \\
\hline $\mathrm{C} 1-\mathrm{C} 2-\mathrm{C} 7-\mathrm{C} 6$ & $179.3(3)$ \\
\hline $\mathrm{C} 2-\mathrm{C} 3-\mathrm{C} 4-\mathrm{C} 5$ & $0.1(7)$ \\
\hline
\end{tabular}

$-0.4(6)$

$-0.3(5)$

$178.8(3)$

$-179.2(3)$

$0.1(6)$

$-1.1(5)$

$-0.7(7)$

$0.4(7)$

$0.5(6)$

$0.8(5)$

$-176.6(3)$

$178.2(3)$

$-1.1(6)$

$1.6(5)$

$0.7(6)$

$0.9(6)$

$-2.0(5)$

$-0.1(5)$

$179.5(2)$

$-179.2(2)$

$-179.3(2)$

$-0.6(3)$

$-0.1(4)$

$-9.3(3)$

$170.6(2)$

0.5 (4)

$-0.2(4)$

$179.2(2)$

$172.0(2)$

$-8.1(4)$

$0.0(4)$

$0.4(3)$

Hydrogen-bond geometry $\left(A,{ }^{\circ}\right)$

$\mathrm{Cg} 1$ and $\mathrm{Cg} 3$ are the centroids of the $\mathrm{C} 2-\mathrm{C} 7$ and $\mathrm{C} 16-\mathrm{C} 21$ rings, respectively.

\begin{tabular}{lllll}
\hline$D-\mathrm{H} \cdots A$ & $D-\mathrm{H}$ & $\mathrm{H} \cdots A$ & $D \cdots A$ & $D-\mathrm{H} \cdots A$ \\
\hline $\mathrm{O} 8-\mathrm{H} 8 A \cdots \mathrm{O} 7^{\mathrm{i}}$ & $0.83(2)$ & $1.75(2)$ & $2.574(2)$ & $168(3)$ \\
$\mathrm{O} 8-\mathrm{H} 8 B \cdots \mathrm{O} 5$ & $0.84(2)$ & $1.76(2)$ & $2.585(3)$ & $166(3)$ \\
$\mathrm{O} 9-\mathrm{H} 9 A \cdots \mathrm{O} 12 A^{\mathrm{ii}}$ & $0.83(2)$ & $2.22(2)$ & $2.988(2)$ & $156(3)$ \\
$\mathrm{O} 9-\mathrm{H} 9 A \cdots \mathrm{O} 12 B^{\mathrm{ii}}$ & $0.83(2)$ & $2.09(2)$ & $2.888(2)$ & $162(3)$ \\
$\mathrm{O} 9-\mathrm{H} 9 B \cdots 6^{i i}$ & $0.83(2)$ & $1.81(2)$ & $2.633(2)$ & $170(3)$ \\
$\mathrm{N} 1-\mathrm{H} 1 A \cdots 6^{\mathrm{i}}$ & $0.88(2)$ & $2.14(2)$ & $3.012(3)$ & $172(2)$ \\
$\mathrm{N} 1-\mathrm{H} 1 B \cdots 6^{\mathrm{ii}}$ & $0.88(2)$ & $2.14(2)$ & $2.965(3)$ & $156(3)$ \\
$\mathrm{N} 2-\mathrm{H} 2 \cdots \mathrm{O} 11 A^{\mathrm{i}}$ & $0.87(2)$ & $2.22(3)$ & $2.936(2)$ & $140(2)$ \\
$\mathrm{N} 2-\mathrm{H} 2 \cdots \mathrm{O} 12 B^{\mathrm{i}}$ & $0.87(2)$ & $2.10(2)$ & $2.946(3)$ & $164(3)$ \\
$\mathrm{N} 3-\mathrm{H} 3 B \cdots \mathrm{O} 4^{4 i}$ & $0.88(2)$ & $2.53(2)$ & $3.344(3)$ & $155(3)$
\end{tabular}


supporting information

$\begin{array}{lllll}\mathrm{N} 4-\mathrm{H} 4 \cdots \mathrm{O} 10 B & 0.87(2) & 2.18(2) & 3.040(3) & 176(3) \\ \mathrm{N} 4-\mathrm{H} 4 \cdots \mathrm{O} 12 A & 0.87(2) & 2.31(3) & 2.970(2) & 133(3) \\ \mathrm{N} 5-\mathrm{H} 5 A \cdots \mathrm{O} 5^{\text {iii }} & 0.88(2) & 2.07(2) & 2.878(3) & 152(2) \\ \mathrm{N} 5-\mathrm{H} 5 B \cdots \mathrm{O} 5 & 0.87(2) & 2.67(2) & 3.199(3) & 120(2) \\ \mathrm{N} 5-\mathrm{H} 5 B \cdots \mathrm{O} 7^{\text {iv }} & 0.87(2) & 2.15(2) & 2.940(3) & 151(2) \\ \mathrm{N} 6-\mathrm{H} 6 \cdots \mathrm{O} 10 A^{\mathrm{v}} & 0.87(2) & 2.22(3) & 2.961(2) & 142(2) \\ \mathrm{N} 6-\mathrm{H} 6 \cdots \mathrm{O} 11 B^{\mathrm{v}} & 0.87(2) & 2.28(2) & 3.115(3) & 159(3) \\ \mathrm{C} 10-\mathrm{H} 10 \cdots \mathrm{O} 10 B & 0.93 & 2.36 & 3.282(2) & 173 \\ \mathrm{C} 11-\mathrm{H} 11 \cdots C g 1^{\mathrm{vi}} & 0.93 & 3.10 & 3.866(2) & 137 \\ \mathrm{C} 13-\mathrm{H} 13 \cdots C g 3^{\mathrm{vii}} & 0.93 & 3.02 & 3.712(4) & 132\end{array}$

Symmetry codes: (i) $x, y-1, z$; (ii) $-x+1,-y+1,-z+1$; (iii) $-x+2,-y,-z+1$; (iv) $-x+2,-y+1,-z+1$; (v) $x+1, y-1, z$; (vi) $x-1, y+1, z$; (vii) $x-1, y, z$. 